\title{
Perceptions on the Competence of Science Teachers along Instruction in a State University in the Philippines
}

\author{
Jomar L. Aban*, Anny Ross A. Bayan, Joan S. Valdez \\ College of Education, Don Mariano Marcos Memorial State University, Bacnotan, La Union 2515, Philippines
}

Received September 1, 2020; Revised October 28, 2020; Accepted November 11, 2020

\section{Cite This Paper in the following Citation Styles}

(a): [1] Jomar L. Aban, Anny Ross A. Bayan, Joan S. Valdez, "Perceptions on the Competence of Science Teachers along Instruction in a State University in the Philippines, "Universal Journal of Educational Research, Vol. 8, No. 12, pp. 6722 - 6736, 2020. DOI: 10.13189/ujer.2020.081237.

(b): Jomar L. Aban, Anny Ross A. Bayan, Joan S. Valdez (2020). Perceptions on the Competence of Science Teachers along Instruction in a State University in the Philippines. Universal Journal of Educational Research, 8(12), 6722 - 6736. DOI: 10.13189/ujer.2020.081237.

Copyright $\odot 2020$ by authors, all rights reserved. Authors agree that this article remains permanently open access under the terms of the Creative Commons Attribution License 4.0 International License

\begin{abstract}
This study hinges on the theory of evaluation. Frequent, continuous and impartial evaluation of academic performance is vital not only for the growth of the institution but also for the growth of the individual. The perceptions of students, teachers and supervisors were evaluated to determine the competence of science teacher along instruction in Don Mariano Marcos Memorial State University (DMMMSU-NLUC), College of Education, Philippines. This research utilized a mixed descriptive-quantitative method of investigation. Using a structured survey questionnaire, the demographic profile and competence of the science teachers were measured. It was found that science teachers with less than ten training points significantly reduced their competence in terms of their personal character, teaching efficiency, general class atmosphere, and substantiality of teaching and evaluation skills. Other personal and professional demographic profiles were also found to have an influence on the competence of science teachers along instruction. These include the number of children, sex, educational attainment, length of service and workload preparation. In conclusion, it is apparent the science teachers' competence is affected by both demographic background of the educators and their personal and professional trainings. This study reveals that knowledge of specific factors that affect science teachers' competence is necessary in improving the mode of instruction, pedagogy and trainings for science teachers to ensure quality teaching.
\end{abstract}

Keywords Science Instruction, Teachers' Competence, Students' Performance, DMMMSU

\section{Introduction}

Teaching or academic instruction is indeed a challenging endeavor for anyone who is ready to face it. One should not depend only on the knowledge he obtained from college but he must have the burning desire to continue growing professionally so that he will be more equipped with the necessary skills, knowledge and attitudes he needs in his profession. Remesh[1] reiterated that as the teacher goes up the educational ladder, his teaching skills are honed in the process. Thus, the higher is the educational attainment of the teacher, the more skillful he becomes in teaching. This was supported by Goldhaber and Brewer[2] revealing that the more matured the teachers were, the better was their performance and the higher the educational attainment of teachers, the better was their performance. This means that competence is enhanced when the teacher goes back to school for higher degrees. In contrary, Bonney et al.[3] inferred that faculty members who have stayed in school for a long time seem to exhibit a declining level of performance in the job and asserted that teachers with a smaller number of years in teaching can make up their experiences with their enthusiasm and eagerness to perform well.

In relation to this, science instruction has a decade-long and persistent history using 'inquiry' as the central word used to characterize good science teaching and learning as perceived by the National Science Education Standards 
(NSES) [4]. Researchers' pursuit of answers in the so-called science inquiry has resulted in an extensive literature. Defining the arena broadly, the number of studies could be in the hundreds and probably more [5]. Inquiry, however, is not the only strategy which can be employed for an effective science instruction. According to Aban et al. [6], generally speaking, in the collegiate instruction from which science instruction is a part, it has to be noted that both instructors and students are stakeholders. Thus, when evaluating the efficiency and effectiveness of different educational pedagogies, these two stakeholders must be recognized. First the instructors: Some instructors may have been teaching for decades and some are just beginning to set their feet flat on the teaching profession. With these differences, come various sorts of pedagogical styles. Some instructors prefer to do lecture method. Some would like their students to be involved in the classroom activities. Others often require students to submit projects and research outputs by the end of the semester. On the other hand, the students also create an impact in science instruction. Instructional strategies employed affect much of the students' grades, attentiveness, interests, outputs and outcomes on a given subject matter.

Furthermore, science instruction may even be affected by various factors including the socio-economic information of science teachers, both personal and professional. Personal profile which includes civil status, sex and number of children may affect competence and efficiency in teaching. Likewise, professional profile including their highest educational attainment, length of service, trainings and workloads may also have an impact on the manner by which they teach in class, their teaching efficiency and the substance of what they are teaching. Hence, this study has been conducted to evaluate these factors and how they affect students' performance in science.

This study aimed to determine the level of competence of science teachers along instruction as perceived by the science teachers themselves, the supervisors of the science teachers and the science students. Specifically, it answered to the following questions: (1) What is the level of competence of science teachers along instruction in terms of: teacher's personal character, teaching efficiency, general class atmosphere, substantiality in teaching, and evaluation skills. (2) Is there a significant difference in the level of competence of Science teachers along instruction when grouped according to various demographic data?

\section{Methodology}

\section{Research Design}

This research study utilized a mixed descriptive-quantitative method of investigation.
According to Nebeker [7], a descriptive study is one in which information is collected without changing the environment. On the other hand, Babbie [8] mentioned that a quantitative approach deals in numbers, logic and an objective stance. The main point of the quantitative research method is that measurement is valid, reliable and can be generalized with its clear anticipation of cause and effect [9]. The descriptive method is also best fitted to be combined with the quantitative method because it involves analyzing, interpreting and elucidating empirical data gathered to give due explanation to numerical figures obtained.

\section{Sources of Data}

The study was conducted at the Don Mariano Marcos Memorial State University - North La Union Campus, College of Education, Bacnotan, La Union. Total population sampling was used in this study. This sampling technique is a type of purposive sampling technique that involves examining the entire population that have a particular set of characteristics. For this research, the respondents are teachers and students involved in science education. There are three types of respondents in this study: (1) the science teachers - these respondents are considered as the self-evaluators. All the nine science teachers in the College of Education - Secondary Education Department were taken as respondents; (2) science students - these are the secondary education students who have basic and core science subjects in the school year 2016 - 2017. A total of 14 students participated as respondents in this study; (3) supervisors - these are the administrators in the College of Education which included the dean, the two chairpersons and the principal who were considered as supervisor evaluators. In total, there are 27 respondents in the study.

\section{Instrumentation and Validity - Reliability Testing}

For this study, a structured survey questionnaire was used as the main tool for gathering most of the information. In addition, thorough interpretation was also used to support the data collected from the questionnaires used. The questionnaire was taken from the study of Cristobal [10] on the competence of teachers along instruction. The questionnaire includes the evaluation of the level of competence of science teachers. Specifically, questions to assess the teacher's personal character, teaching efficiency, general class atmosphere, substantiality of teaching and evaluation skills were asked and interpreted.

Another tool that supported this questionnaire was the Teaching and Learning International Survey (TALIS) Teacher Questionnaire by the International Project Consortium: International Association for the Evaluation of Educational Achievement (IEA), The Netherlands IEA Data Processing and Research Center (IEA DPC). Based 
on the matter by which the survey questionnaire was built and how it was previously used, the validity and the reliability of the survey questionnaire is assumed.

\section{Data Presentation and Statistical Analysis}

The IBM SPSS Statistics Version 20 was used for all data presentation and statistical analysis performed in this study. The different level of teacher competence (teacher's personal character, teaching efficiency, general class atmosphere, substantiality in teaching, and evaluation skills) were grouped according to the different personal and professional demographic data of the respondents. These were presented in a table using mean scores with \pm standard deviation. Their descriptive equivalent was also projected together with the F-crit and significance. This was done by comparing the means of the dependent and independent variables. Using the same software, one-way Analysis of Variance (ANOVA) with Scheffe post-hoc test was used to determine significant differences between the means of those competence parameters with three or more independent groups. The following scale is used to categorize the competence of teachers: $3.26-4.00$ is Highly Competent; $2.51-3.25$ is Competent; $1.76-2.50$ is Fairly Competent; and 1.00-1.75 is Not Competent.

\section{Results and Discussion}

The difference criteria pertaining to the level of competence of the science teachers were presented in the succeeding tables. These were grouped according to the different personal data as well as the professional demographics of the faculty members. This included (1) teacher's personal characteristics; (2) teaching efficiency; (3) general atmosphere in class; (4) substantiality of teaching; and (5) evaluation skills. For the teacher's personal characteristics, the criteria would include the clarity and modulation of voice, the maintenance of self-control both in action and words as well as the teacher's fluency and explanation of the lesson using the language of instruction. For teaching efficiency, it was gauged according to the effectiveness of methods and techniques of teaching as well as the motivation for learning and critical reflection. For the general atmosphere in class, this was the measure on how conducive is the classroom for learning and how the teacher maintains discipline and implements school policies among students. For the substantiality of teaching, it measured the teacher's mastery of subject matter and how he presents the important learning aspects to gain in-depth knowledge of the subject matter. Lastly, for the evaluation skills, it determined how the teacher asks thought provoking questions and how well he prepares well-framed test questions that are valid and reliable.

Table 1 presents the teachers' personal characteristics as grouped according to demographic data. When grouped according to sex, both male and female teachers are highly competent, although female seemed to have higher competence as perceived by the three types of evaluators. The results are also comparable when the respondents were grouped according to civil status and the number of children. Similar not significant results can also be observed for both the educational attainment and the length of service of the respondents. However, it can be gleaned that as the respondents attained higher degree and longer length of service an increasing level of competence can be observed. Previous studies have suggested that a teacher's personal character is affected by their educational achievement and length of service. According to Shah[11], the higher the educational attainment of a teacher, the more he develops his personal character which leads to university students' academic achievement. In addition, Wenglinsky[12] said that the length of service of a teacher also molds their character and behavior, thus, a better personal teaching characteristic is offered by teacher that is longer in service.

Table 2 presents teachers' personal characteristics as affected by trainings and workloads. It can be observed from the table that respondents with less than 10 training points are less competent $(3.15 \pm 32)$ than those with higher training points $(11-20 \mathrm{pts} .=3.81 \pm 31 ;>20 \mathrm{pts} .=3.85 \pm .23)$ as perceived by student respondents. An increasing competence level can also be observed as the number of trainings increased. For the teaching units, it can be observed that teachers with higher than 21 teaching units seemed to have lower competence $(3.25 \pm 49)$ compared to those with a smaller number of teaching loads $(3.71 \pm 49)$ as rated by student respondents. The number of preparations may also affect the competence of science teachers since it can be gleaned from the table that those teachers with more than 5 preparations have lesser personal character competence $(3.24 \pm 53)$ compared to those with less subject preparations. A decreasing competence can also be observed as the number of preparations increases. The reason for this observed result is the mastery of content. Teachers who are asked to prepare more than five subjects to teach, would require more time to master each of these subjects compared to those teachers who only have one or two subject preparations. On the other hand, no significant impact to teacher's personal character when it comes to the number of quasi assignments. 
Table 1. Teachers' personal character as grouped according to demographic data

\begin{tabular}{|c|c|c|c|c|c|c|}
\hline Type of Evaluation & Variable & Mean & S.D. & Descriptive Equivalent & $\mathrm{F}$ & Sig. \\
\hline \multicolumn{7}{|c|}{ Sex } \\
\hline Self-Evaluation & $\begin{array}{c}\text { Male } \\
\text { Female }\end{array}$ & $\begin{array}{l}3.62^{\mathrm{a}} \\
3.85^{\mathrm{a}} \\
\end{array}$ & $\begin{array}{l} \pm .51 \\
\pm .53 \\
\end{array}$ & $\begin{array}{l}\text { Highly Competent } \\
\text { Highly Competent } \\
\end{array}$ & .078 & $.783^{\mathrm{ns}}$ \\
\hline Student Evaluation & $\begin{array}{l}\text { Male } \\
\text { Female }\end{array}$ & $\begin{array}{l}3.44^{\mathrm{a}} \\
3.62^{\mathrm{a}}\end{array}$ & $\begin{array}{l}+.49 \\
+.53\end{array}$ & $\begin{array}{l}\text { Highly Competent } \\
\text { Highly Competent }\end{array}$ & .174 & $.680^{\mathrm{ns}}$ \\
\hline Supervisor Evaluation & $\begin{array}{c}\text { Male } \\
\text { Female }\end{array}$ & $\begin{array}{l}3.88^{\mathrm{a}} \\
4.00^{\mathrm{a}} \\
\end{array}$ & $\begin{array}{l}+.50 \\
+55 \\
\end{array}$ & $\begin{array}{l}\text { Highly Competent } \\
\text { Highly Competent }\end{array}$ & .103 & $.751^{\mathrm{ns}}$ \\
\hline \multicolumn{7}{|c|}{ Civil Status } \\
\hline Self-Evaluation & $\begin{array}{c}\text { Single } \\
\text { Married }\end{array}$ & $\begin{array}{l}3.67^{\mathrm{a}} \\
3.91^{\mathrm{a}}\end{array}$ & $\begin{array}{r}+.53 \\
+.50\end{array}$ & $\begin{array}{l}\text { Highly Competent } \\
\text { Highly Competent }\end{array}$ & .014 & $.905^{\mathrm{ns}}$ \\
\hline Student Evaluation & $\begin{array}{l}\text { Single } \\
\text { Married }\end{array}$ & $\begin{array}{l}3.45^{\mathrm{a}} \\
3.69^{\mathrm{a}}\end{array}$ & $\begin{array}{l}+.52 \\
+.50\end{array}$ & $\begin{array}{l}\text { Highly Competent } \\
\text { Highly Competent }\end{array}$ & .005 & $.942^{\mathrm{ns}}$ \\
\hline Supervisor Evaluation & $\begin{array}{l}\text { Single } \\
\text { Married }\end{array}$ & $\begin{array}{l}3.85^{\mathrm{a}} \\
4.00^{\mathrm{a}} \\
\end{array}$ & $\begin{array}{l} \pm .52 \\
+.50 \\
\end{array}$ & $\begin{array}{l}\text { Highly Competent } \\
\text { Highly Competent }\end{array}$ & .005 & $.942^{\mathrm{ns}}$ \\
\hline \multicolumn{7}{|c|}{ No. of Children } \\
\hline Self-Evaluation & $\begin{array}{l}\text { None } \\
\text { One } \\
\text { Three }\end{array}$ & $\begin{array}{l}3.67^{\mathrm{a}} \\
3.96^{\mathrm{a}} \\
3.74^{\mathrm{a}}\end{array}$ & $\begin{array}{l} \pm .42 \\
\pm .38 \\
+.41\end{array}$ & $\begin{array}{l}\text { Highly Competent } \\
\text { Highly Competent } \\
\text { Highly Competent }\end{array}$ & .858 & $.436^{\mathrm{ns}}$ \\
\hline Student Evaluation & $\begin{array}{l}\text { None } \\
\text { One } \\
\text { Three }\end{array}$ & $\begin{array}{l}3.45^{\mathrm{a}} \\
3.75^{\mathrm{a}} \\
3.52^{\mathrm{a}}\end{array}$ & $\begin{array}{l} \pm .47 \\
+.50 \\
+.39\end{array}$ & $\begin{array}{l}\text { Highly Competent } \\
\text { Highly Competent } \\
\text { Highly Competent }\end{array}$ & .921 & $.412^{\mathrm{ns}}$ \\
\hline Supervisor Evaluation & $\begin{array}{l}\text { None } \\
\text { One } \\
\text { Three }\end{array}$ & $\begin{array}{l}3.85^{\mathrm{a}} \\
4.00^{\mathrm{a}} \\
3.98^{\mathrm{a}} \\
\end{array}$ & $\begin{array}{l} \pm .42 \\
\pm .39 \\
+.43 \\
\end{array}$ & $\begin{array}{l}\text { Highly Competent } \\
\text { Highly Competent } \\
\text { Highly Competent }\end{array}$ & 1.64 & $.215^{\mathrm{ns}}$ \\
\hline \multicolumn{7}{|c|}{ Highest Educational Attainment } \\
\hline Self-Evaluation & $\begin{array}{c}\text { BS degree } \\
\text { MS units } \\
\text { PhD degree }\end{array}$ & $\begin{array}{l}3.62^{\mathrm{a}} \\
3.73^{\mathrm{a}} \\
3.90^{\mathrm{a}}\end{array}$ & $\begin{array}{l}+.20 \\
\pm .28 \\
+.21\end{array}$ & $\begin{array}{l}\text { Highly Competent } \\
\text { Highly Competent } \\
\text { Highly Competent }\end{array}$ & .744 & $.486^{\mathrm{ns}}$ \\
\hline Student Evaluation & $\begin{array}{l}\text { BS degree } \\
\text { MS units } \\
\text { PhD degree }\end{array}$ & $\begin{array}{l}3.42^{\mathrm{a}} \\
3.49^{\mathrm{a}} \\
3.69^{\mathrm{a}}\end{array}$ & $\begin{array}{l}+.32 \\
+.29 \\
+.30\end{array}$ & $\begin{array}{l}\text { Highly Competent } \\
\text { Highly Competent } \\
\text { Highly Competent }\end{array}$ & .734 & $.490^{\mathrm{ns}}$ \\
\hline Supervisor Evaluation & $\begin{array}{l}\text { BS degree } \\
\text { MS units } \\
\text { PhD degree }\end{array}$ & $\begin{array}{l}3.82^{\mathrm{a}} \\
3.89^{\mathrm{a}} \\
4.19^{\mathrm{a}}\end{array}$ & $\begin{array}{l} \pm .23 \\
\pm .21 \\
\pm .38 \\
\end{array}$ & $\begin{array}{l}\text { Highly Competent } \\
\text { Highly Competent } \\
\text { Highly Competent }\end{array}$ & 1.44 & $.256^{\mathrm{ns}}$ \\
\hline \multicolumn{7}{|c|}{ Length of Service } \\
\hline Self-Evaluation & $\begin{array}{c}<5 \text { years } \\
5-9 \text { years } \\
10-14 \text { years }\end{array}$ & $\begin{array}{l}3.67^{\mathrm{a}} \\
3.74^{\mathrm{a}} \\
4.00^{\mathrm{a}}\end{array}$ & $\begin{array}{l}+.31 \\
+.28 \\
+.33\end{array}$ & $\begin{array}{l}\text { Highly Competent } \\
\text { Highly Competent } \\
\text { Highly Competent }\end{array}$ & 1.27 & $.300^{\mathrm{ns}}$ \\
\hline Student Evaluation & $\begin{array}{c}<5 \text { years } \\
5-9 \text { years } \\
10-14 \text { years }\end{array}$ & $\begin{array}{l}3.46^{\mathrm{a}} \\
3.53^{\mathrm{a}} \\
3.86^{\mathrm{a}}\end{array}$ & $\begin{array}{l}+.22 \\
+.25 \\
+.34\end{array}$ & $\begin{array}{l}\text { Highly Competent } \\
\text { Highly Competent } \\
\text { Highly Competent }\end{array}$ & 1.31 & $.289^{\mathrm{ns}}$ \\
\hline Supervisor Evaluation & $\begin{array}{c}5 \text { years } \\
5-9 \text { years } \\
10-14 \text { years } \\
\end{array}$ & $\begin{array}{l}3.88^{\mathrm{a}} \\
3.92^{\mathrm{a}} \\
4.00^{\mathrm{a}}\end{array}$ & $\begin{array}{l}+.22 \\
\pm .23 \\
\pm .23 \\
+.29 \\
\end{array}$ & $\begin{array}{l}\text { Highly Competent } \\
\text { Highly Competent } \\
\text { Highly Competent }\end{array}$ & 1.90 & $.171^{\mathrm{ns}}$ \\
\hline
\end{tabular}

Means with different letters are significantly different $(\rho=0.05)$. Values are expressed in mean \pm S.D. Symbols and abbreviations: $* *=$ highly significant; $*=$ significant; $\mathrm{ns}=$ not significant

There were recent reports indicating that trainings can affect a teacher's personal character. In the study of Dolev and Leshem [13], they have reported that a teacher's emotional intelligence or their personal character is affected by teacher trainings. Despite the necessity, Jennings and Greenberg [14] reported that there has been little focus on teacher's own development through training. This ambiguity should therefore be addressed. Teacher trainings, such as those related to the teaching and learning process, recognize the importance of improving personal character of a teacher [15] which in turn increases emotional information of both own emotions and those students in lesson plans and in the classroom [16].
Furthermore, trainings enhance teachers' sensitivity to students' emotions in different situations [17], they also enhance teachers' ability to respond constructively to students' social-emotional needs [18]. This present study therefore promotes the need for teachers to be involved in contemporary trainings and seminars for their professional teaching development. There are a few published literature concerning the impact of teaching units and number of preparations on the personal character of a teacher. In the study of Wilson et al. [19], they have conducted a review regarding questions concerning teacher preparation. Since teaching units and number of preparations are considered workload of teachers, increasing the number of these 
parameters would certainly affect a teacher's behavior in the classroom. Physical and emotional fatigue due to effort exerted by teachers on their additional teaching units and preparations would lead to emotional stress, insensitivity and failure to show constructive ways of criticism.

The teaching efficiency as grouped according to demographic data is presented in Table 3. For both sex and civil status, no significant differences can be observed. This means regardless of sex and civil status; the respondents were found to be highly competent in terms of their efficiency in teaching. However, the number of children seemed to have a consequence to teaching efficiency. Based on the above results, those respondents with the highest number of children appeared to less competent $(3.20 \pm .55)$ in their teaching efficiency. This result is, however, not statistically significant. Nonetheless, the numerical results imply that the higher the number of children raised by the teacher, the lesser competent he/she becomes. This can potentially be caused by issues arising from personal versus professional time management, where a teacher's time is divided either proportionately or disproportionately based on his/her priority. On the other hand, comparable efficiency in teaching can be observed when grouped according to respondents' highest educational attainment. But, as per data presented above, the length of service influences teaching efficiency, where those respondents with the shortest teaching experience (3.24 \pm 51$)$ appeared to have lower competence than those with longer length of service.

Table 2. Teacher's personal character as affected by trainings and workloads

\begin{tabular}{|c|c|c|c|c|c|c|}
\hline Type of Evaluation & Variable & Mean & S.D. & Descriptive Equivalent & $\mathrm{F}$ & Sig. \\
\hline \multicolumn{7}{|c|}{ Training Points } \\
\hline Self-Evaluation & $\begin{array}{l}<10 \text { pts. } \\
11-20 \text { pts. } \\
>20 \text { pts. }\end{array}$ & $\begin{array}{l}3.39^{\mathrm{a}} \\
3.74^{\mathrm{a}} \\
3.90^{\mathrm{a}} \\
\end{array}$ & $\begin{array}{l} \pm .20 \\
\pm .21 \\
+.31 \\
\end{array}$ & $\begin{array}{l}\text { Highly Competent } \\
\text { Highly Competent } \\
\text { Highly Competent }\end{array}$ & .809 & $.590^{\mathrm{ns}}$ \\
\hline Student Evaluation & $\begin{array}{l}<\mathbf{1 0} \text { pts. } \\
11-20 \text { pts. } \\
>20 \text { pts. }\end{array}$ & $\begin{array}{l}3.15^{\mathrm{b}} \\
3.81^{\mathrm{a}} \\
3.85^{\mathrm{a}} \\
\end{array}$ & $\begin{array}{l}+.32 \\
\pm .31 \\
\pm .38 \\
+.28\end{array}$ & $\begin{array}{c}\text { Competent } \\
\text { Highly Competent } \\
\text { Highly Competent }\end{array}$ & 4.33 & $.041^{*}$ \\
\hline Supervisor Evaluation & $\begin{array}{c}<10 \text { pts. } \\
11-20 \text { pts. } \\
>20 \text { pts. }\end{array}$ & $\begin{array}{l}3.55^{\mathrm{a}} \\
3.70^{\mathrm{a}} \\
3.89^{\mathrm{a}} \\
\end{array}$ & $\begin{array}{l}.23 \\
\pm .40 \\
+.33 \\
\end{array}$ & $\begin{array}{l}\text { Highly Competent } \\
\text { Highly Competent } \\
\text { Highly Competent } \\
\end{array}$ & .898 & $.528^{\mathrm{ns}}$ \\
\hline \multicolumn{7}{|c|}{ Teaching Units } \\
\hline Self-Evaluation & $\begin{array}{l}<20 \text { units } \\
>21 \text { units }\end{array}$ & $\begin{array}{l}3.93^{\mathrm{a}} \\
3.57^{\mathrm{a}} \\
\end{array}$ & $\begin{array}{l} \pm .49 \\
\pm .50 \\
\end{array}$ & $\begin{array}{l}\text { Highly Competent } \\
\text { Highly Competent }\end{array}$ & 3.61 & $.069^{\text {ns }}$ \\
\hline Student Evaluation & $\begin{array}{l}<20 \text { units } \\
>21 \text { units }\end{array}$ & $\begin{array}{l}3.71^{\mathrm{a}} \\
\mathbf{3 . 2 5 ^ { \mathrm { b } }}\end{array}$ & $\begin{array}{l} \pm .49 \\
\pm .49 \\
\end{array}$ & $\begin{array}{c}\text { Highly Competent } \\
\text { Competent }\end{array}$ & 5.19 & $.032^{*}$ \\
\hline Supervisor Evaluation & $\begin{array}{l}<20 \text { units } \\
>21 \text { units } \\
\end{array}$ & $\begin{array}{l}3.98^{\mathrm{a}} \\
3.76^{\mathrm{a}} \\
\end{array}$ & $\begin{array}{l}+.49 \\
+.50 \\
\end{array}$ & $\begin{array}{l}\text { Highly Competent } \\
\text { Highly Competent }\end{array}$ & 3.43 & $.076^{\mathrm{ns}}$ \\
\hline \multicolumn{7}{|c|}{ Number of Preparations } \\
\hline Self-Evaluation & $\begin{array}{c}2 \text { Prep } \\
2-4 \text { Preps } \\
>5 \text { Preps } \\
\end{array}$ & $\begin{array}{l}3.84^{\mathrm{a}} \\
3.74^{\mathrm{a}} \\
3.57^{\mathrm{a}} \\
\end{array}$ & $\begin{array}{l}+.55 \\
+.53 \\
+.52 \\
\end{array}$ & $\begin{array}{l}\text { Highly Competent } \\
\text { Highly Competent } \\
\text { Highly Competent } \\
\end{array}$ & .623 & $.545^{\mathrm{ns}}$ \\
\hline Student Evaluation & $\begin{array}{c}2 \text { Prep } \\
\text { 2-4 Preps } \\
>5 \text { Preps }\end{array}$ & $\begin{array}{l}3.64^{\mathrm{a}} \\
3.52^{\mathrm{a}} \\
\mathbf{3 . 2 4 ^ { \mathrm { b } }}\end{array}$ & $\begin{array}{l} \pm .55 \\
\pm .51 \\
+.53\end{array}$ & $\begin{array}{l}\text { Highly Competent } \\
\text { Highly Competent } \\
\text { Competent }\end{array}$ & 7.08 & $.050^{*}$ \\
\hline Supervisor Evaluation & $\begin{array}{c}2 \text { Prep } \\
2-4 \text { Preps } \\
>5 \text { Preps } \\
\end{array}$ & $\begin{array}{l}3.98^{\mathrm{a}} \\
3.97^{\mathrm{a}} \\
3.90^{\mathrm{a}} \\
\end{array}$ & $\begin{array}{l}+.55 \\
\pm .53 \\
+.56 \\
\end{array}$ & $\begin{array}{l}\text { Highly Competent } \\
\text { Highly Competent } \\
\text { Highly Competent }\end{array}$ & .587 & $.564^{\mathrm{ns}}$ \\
\hline \multicolumn{7}{|c|}{ Number of Quasi Assignments } \\
\hline Self-Evaluation & $\begin{array}{l}<2 \text { Quasi } \\
2-4 \text { Quasi } \\
>5 \text { Quasi } \\
\end{array}$ & $\begin{array}{c}3.74^{\mathrm{a}} \\
3.77^{\mathrm{a}} \\
\mathrm{n} / \mathrm{a} \\
\end{array}$ & $\begin{array}{c} \pm .55 \\
\pm .53 \\
\mathrm{n} / \mathrm{a} \\
\end{array}$ & $\begin{array}{c}\text { Highly Competent } \\
\text { Highly Competent } \\
\mathrm{n} / \mathrm{a} \\
\end{array}$ & .014 & $.907^{\mathrm{ns}}$ \\
\hline Student Evaluation & $\begin{array}{l}<2 \text { Quasi } \\
2-4 \text { Quasi } \\
>5 \text { Quasi }\end{array}$ & $\begin{array}{c}3.51^{\mathrm{a}} \\
3.56^{\mathrm{a}} \\
\mathrm{n} / \mathrm{a}\end{array}$ & $\begin{array}{c} \pm .55 \\
\pm .52 \\
\mathrm{n} / \mathrm{a} \\
\end{array}$ & $\begin{array}{c}\text { Highly Competent } \\
\text { Highly Competent } \\
\mathrm{n} / \mathrm{a} \\
\end{array}$ & .036 & $.851^{\mathrm{ns}}$ \\
\hline Supervisor Evaluation & $\begin{array}{l}<2 \text { Quasi } \\
2-4 \text { Quasi } \\
>5 \text { Quasi }\end{array}$ & $\begin{array}{c}3.90^{\mathrm{a}} \\
3.93^{\mathrm{a}} \\
\mathrm{n} / \mathrm{a} \\
\end{array}$ & $\begin{array}{c} \pm .55 \\
\pm .54 \\
\mathrm{n} / \mathrm{a} \\
\end{array}$ & $\begin{array}{c}\text { Highly Competent } \\
\text { Highly Competent } \\
\text { n/a }\end{array}$ & .113 & $.739^{\mathrm{ns}}$ \\
\hline
\end{tabular}


Table 3. Teaching efficiency as grouped according to different demographic data

\begin{tabular}{|c|c|c|c|c|c|c|}
\hline Type of Evaluation & Variable & Mean & S.D. & Descriptive Equivalent & $\mathrm{F}$ & Sig. \\
\hline \multicolumn{7}{|c|}{ Sex } \\
\hline Self-Evaluation & $\begin{array}{l}\text { Male } \\
\text { Female }\end{array}$ & $\begin{array}{l}3.58^{\mathrm{a}} \\
3.73^{\mathrm{a}}\end{array}$ & $\begin{array}{l}+.62 \\
\pm .52\end{array}$ & $\begin{array}{l}\text { Highly Competent } \\
\text { Highly Competent }\end{array}$ & .406 & $.530^{\mathrm{ns}}$ \\
\hline Student Evaluation & $\begin{array}{c}\text { Male } \\
\text { Female }\end{array}$ & $\begin{array}{l}3.40^{\mathrm{a}} \\
3.50^{\mathrm{a}} \\
\end{array}$ & $\begin{array}{l}+.58 \\
+.52 \\
\end{array}$ & $\begin{array}{l}\text { Highly Competent } \\
\text { Highly Competent }\end{array}$ & .205 & $.654^{\text {ns }}$ \\
\hline Supervisor Evaluation & $\begin{array}{c}\text { Male } \\
\text { Female }\end{array}$ & $\begin{array}{l}3.84^{\mathrm{a}} \\
3.95^{\mathrm{a}} \\
\end{array}$ & $\begin{array}{r}+.59 \\
+.52 \\
\end{array}$ & $\begin{array}{l}\text { Highly Competent } \\
\text { Highly Competent }\end{array}$ & .273 & $.606^{\mathrm{ns}}$ \\
\hline \multicolumn{7}{|c|}{ Civil Status } \\
\hline Self-Evaluation & $\begin{array}{l}\text { Single } \\
\text { Married }\end{array}$ & $\begin{array}{l}3.64^{\mathrm{a}} \\
3.73^{\mathrm{a}}\end{array}$ & $\begin{array}{l} \pm .60 \\
\pm .49\end{array}$ & $\begin{array}{l}\text { Highly Competent } \\
\text { Highly Competent }\end{array}$ & .161 & $.692^{\text {ns }}$ \\
\hline Student Evaluation & $\begin{array}{l}\text { Single } \\
\text { Married }\end{array}$ & $\begin{array}{l}3.43^{\mathrm{a}} \\
3.52^{\mathrm{a}}\end{array}$ & $\begin{array}{l} \pm .57 \\
+.50\end{array}$ & $\begin{array}{l}\text { Highly Competent } \\
\text { Highly Competent }\end{array}$ & .191 & $.667^{\text {ns }}$ \\
\hline Supervisor Evaluation & $\begin{array}{c}\text { Single } \\
\text { Married }\end{array}$ & $\begin{array}{l}3.83^{\mathrm{a}} \\
3.91^{\mathrm{a}} \\
\end{array}$ & $\begin{array}{l}+.57 \\
\pm .49 \\
\end{array}$ & $\begin{array}{l}\text { Highly Competent } \\
\text { Highly Competent }\end{array}$ & .836 & $.369^{\text {ns }}$ \\
\hline \multicolumn{7}{|c|}{ No. of Children } \\
\hline Self-Evaluation & $\begin{array}{l}\text { None } \\
\text { One } \\
\text { Three }\end{array}$ & $\begin{array}{l}3.64^{\mathrm{a}} \\
3.81^{\mathrm{a}} \\
3.51^{\mathrm{a}}\end{array}$ & $\begin{array}{l} \pm .60 \\
\pm .49 \\
+.55\end{array}$ & $\begin{array}{l}\text { Highly Competent } \\
\text { Highly Competent } \\
\text { Highly Competent }\end{array}$ & .398 & $.676^{\mathrm{ns}}$ \\
\hline Student Evaluation & $\begin{array}{l}\text { None } \\
\text { One } \\
\text { Three }\end{array}$ & $\begin{array}{l}3.43^{\mathrm{a}} \\
3.60^{\mathrm{a}} \\
\mathbf{3 . 2 0 ^ { \mathrm { a } }} \\
\end{array}$ & $\begin{array}{r}+.57 \\
\pm .48 \\
+.55 \\
\end{array}$ & $\begin{array}{c}\text { Highly Competent } \\
\text { Highly Competent } \\
\text { Competent }\end{array}$ & .444 & $.647^{\mathrm{ns}}$ \\
\hline Supervisor Evaluation & $\begin{array}{l}\text { None } \\
\text { One } \\
\text { Three }\end{array}$ & $\begin{array}{l}3.83^{\mathrm{a}} \\
3.93^{\mathrm{a}} \\
3.79^{\mathrm{a}} \\
\end{array}$ & $\begin{array}{l}+.57 \\
+.49 \\
+.49 \\
+.53 \\
\end{array}$ & $\begin{array}{l}\text { Highly Competent } \\
\text { Highly Competent } \\
\text { Highly Competent }\end{array}$ & .763 & $.477^{\text {ns }}$ \\
\hline \multicolumn{7}{|c|}{ Highest Educational Attainment } \\
\hline Self-Evaluation & $\begin{array}{c}\text { BS degree } \\
\text { MS units } \\
\text { PhD degree }\end{array}$ & $\begin{array}{l}3.62^{\mathrm{a}} \\
3.67^{\mathrm{a}} \\
3.73^{\mathrm{a}} \\
\end{array}$ & $\begin{array}{l}+.58 \\
\pm .48 \\
\pm .49\end{array}$ & $\begin{array}{l}\text { Highly Competent } \\
\text { Highly Competent } \\
\text { Highly Competent }\end{array}$ & .093 & $.911^{\mathrm{ns}}$ \\
\hline Student Evaluation & $\begin{array}{l}\text { BS degree } \\
\text { MS units } \\
\text { PhD degree }\end{array}$ & $\begin{array}{l}3.43^{\mathrm{a}} \\
3.43^{\mathrm{a}} \\
3.52^{\mathrm{a}} \\
\end{array}$ & $\begin{array}{l}-.56 \\
\pm .56 \\
\pm .63 \\
\pm .47 \\
\end{array}$ & $\begin{array}{l}\text { Highly Competent } \\
\text { Highly Competent } \\
\text { Highly Competent }\end{array}$ & .092 & $.913^{\text {ns }}$ \\
\hline Supervisor Evaluation & $\begin{array}{l}\text { BS degree } \\
\text { MS units } \\
\text { PhD degree }\end{array}$ & $\begin{array}{l}3.83^{\mathrm{a}} \\
3.84^{\mathrm{a}} \\
3.91^{\mathrm{a}} \\
\end{array}$ & $\begin{array}{l}+.57 \\
\pm .60 \\
\pm .60 \\
+.55 \\
\end{array}$ & $\begin{array}{l}\text { Highly Competent } \\
\text { Highly Competent } \\
\text { Highly Competent }\end{array}$ & .401 & $.674^{\mathrm{ns}}$ \\
\hline \multicolumn{7}{|c|}{ Length of Service } \\
\hline Self-Evaluation & $\begin{array}{c}5 \text { years } \\
5-9 \text { years } \\
10-14 \text { years }\end{array}$ & $\begin{array}{l}3.50^{\mathrm{a}} \\
3.66^{\mathrm{a}} \\
3.84^{\mathrm{a}}\end{array}$ & $\begin{array}{r}+.46 \\
+.44 \\
+.47 \\
\end{array}$ & $\begin{array}{l}\text { Highly Competent } \\
\text { Highly Competent } \\
\text { Highly Competent }\end{array}$ & .399 & $.675^{\mathrm{ns}}$ \\
\hline Student Evaluation & $\begin{array}{c}\mathbf{5} \text { years } \\
5-9 \text { years } \\
10-14 \text { years } \\
\end{array}$ & $\begin{array}{l}.24^{\mathrm{b}} \\
3.45^{\mathrm{a}} \\
3.63^{\mathrm{a}} \\
\end{array}$ & $\begin{array}{l}+.51 \\
\pm .45 \\
\pm .45 \\
+.58 \\
\end{array}$ & $\begin{array}{c}\text { Competent } \\
\text { Highly Competent } \\
\text { Highly Competent }\end{array}$ & 4.28 & $.050^{*}$ \\
\hline Supervisor Evaluation & $\begin{array}{c}5 \text { years } \\
5-9 \text { years } \\
10-14 \text { years } \\
\end{array}$ & $\begin{array}{l}3.79^{\mathrm{a}} \\
3.86^{\mathrm{a}} \\
3.91^{\mathrm{a}} \\
\end{array}$ & $\begin{array}{l}+.61 \\
\pm .47 \\
\pm .50 \\
\end{array}$ & $\begin{array}{l}\text { Highly Competent } \\
\text { Highly Competent } \\
\text { Highly Competent } \\
\end{array}$ & .610 & $.552^{\mathrm{ns}}$ \\
\hline
\end{tabular}

Means with different letters are significantly different $(\rho=0.05)$. Values are expressed in mean \pm S.D. Symbols and abbreviations: $* *=$ highly significant; $*=$ significant; $\mathrm{ns}=$ not significant

Dearth information would reveal that the number of children a teacher has would affect his teaching efficiency. In the study of teaching effectiveness of secondary teachers in relation to their demographic characteristics by Tyagi[20], she pointed out that teaching effectiveness has a relationship between teacher, pupils and other persons concerned with the educational undertaking. As previously mentioned, it is possible that teachers with more children may have the tendency to devote less time to their students since they also need to spend time with their children and their family in general. Although other extraneous variables might still affect their efficiency in teaching, their social background, teaching subjects, qualification and school teaching experiences. On the basis of results obtained, it was inferred that teaching efficiency is affected by the length of teaching service. Wherein, teachers with less than 5 years of teaching experience would likely have lower teaching efficiency than those with longer years of service. This is similar to the results of the study of Centra [21] where he found out that secondary school teachers of above 3 years have higher level of effectiveness in teaching than secondary school teachers having up to 3 years school teaching experience with reference to their knowledge. 
Table 4. Teaching efficiency as affected by trainings and workloads

\begin{tabular}{|c|c|c|c|c|c|c|}
\hline Type of Evaluation & Variable & Mean & S.D. & Descriptive Equivalent & $\mathrm{F}$ & Sig. \\
\hline \multicolumn{7}{|c|}{ Training Points } \\
\hline Self-Evaluation & $\begin{array}{l}<\mathbf{1 0} \text { pts. } \\
11-20 \text { pts. } \\
>20 \text { pts. }\end{array}$ & $\begin{array}{l}\mathbf{3 . 0 6}^{\mathrm{b}} \\
3.67^{\mathrm{a}} \\
3.94^{\mathrm{a}}\end{array}$ & $\begin{array}{l}+.58 \\
\pm .55 \\
+.51\end{array}$ & $\begin{array}{c}\text { Competent } \\
\text { Highly Competent } \\
\text { Highly Competent }\end{array}$ & 8.67 & $.048^{*}$ \\
\hline Student Evaluation & $\begin{array}{l}<\mathbf{1 0} \text { pts. } \\
11-20 \text { pts. } \\
>20 \text { pts. }\end{array}$ & 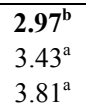 & $\begin{array}{l} \pm .54 \\
\pm .41 \\
+.59\end{array}$ & $\begin{array}{c}\text { Competent } \\
\text { Highly Competent } \\
\text { Highly Competent }\end{array}$ & 6.51 & $0.50^{*}$ \\
\hline Supervisor Evaluation & $\begin{array}{l}<10 \text { pts. } \\
11-20 \text { pts. } \\
>20 \text { pts. }\end{array}$ & $\begin{array}{l}3.37^{\mathrm{a}} \\
3.79^{\mathrm{a}} \\
3.89^{\mathrm{a}} \\
\end{array}$ & $\begin{array}{l}+.39 \\
\pm .47 \\
\pm .40 \\
\end{array}$ & $\begin{array}{l}\text { Highly Competent } \\
\text { Highly Competent } \\
\text { Highly Competent }\end{array}$ & .737 & $.644^{\mathrm{ns}}$ \\
\hline \multicolumn{7}{|c|}{ Teaching Units } \\
\hline Self-Evaluation & $\begin{array}{l}<20 \text { units } \\
>21 \text { units }\end{array}$ & $\begin{array}{l}3.79^{\mathrm{a}} \\
3.53^{\mathrm{a}} \\
\end{array}$ & $\begin{array}{l} \pm .51 \\
+.50 \\
\end{array}$ & $\begin{array}{l}\text { Highly Competent } \\
\text { Highly Competent }\end{array}$ & 1.51 & $.231^{\mathrm{ns}}$ \\
\hline Student Evaluation & $\begin{array}{l}<20 \text { units } \\
>21 \text { units }\end{array}$ & $\begin{array}{l}3.58^{\mathrm{a}} \\
3.33^{\mathrm{a}}\end{array}$ & $\begin{array}{l} \pm .49 \\
\pm .47 \\
\end{array}$ & $\begin{array}{l}\text { Highly Competent } \\
\text { Highly Competent }\end{array}$ & 1.44 & $.240^{\mathrm{ns}}$ \\
\hline Supervisor Evaluation & $\begin{array}{l}<20 \text { units } \\
>21 \text { units }\end{array}$ & $\begin{array}{l}3.91^{\mathrm{a}} \\
3.73^{\mathrm{a}} \\
\end{array}$ & $\begin{array}{l} \pm .51 \\
\pm .41 \\
\end{array}$ & $\begin{array}{l}\text { Highly Competent } \\
\text { Highly Competent }\end{array}$ & 2.58 & $.120^{\mathrm{ns}}$ \\
\hline \multicolumn{7}{|c|}{ Number of Preparations } \\
\hline Self-Evaluation & $\begin{array}{c}<2 \text { Prep } \\
2-4 \text { Preps } \\
>5 \text { Preps } \\
\end{array}$ & $\begin{array}{l}3.73^{\mathrm{a}} \\
3.69^{\mathrm{a}} \\
3.50^{\mathrm{a}} \\
\end{array}$ & $\begin{array}{l}+.55 \\
+.59 \\
+.55 \\
\end{array}$ & $\begin{array}{l}\text { Highly Competent } \\
\text { Highly Competent } \\
\text { Highly Competent }\end{array}$ & .175 & $.841^{\mathrm{ns}}$ \\
\hline Student Evaluation & $\begin{array}{l}<2 \text { Prep } \\
2-4 \text { Preps } \\
>5 \text { Preps }\end{array}$ & $\begin{array}{l}3.51^{\mathrm{a}} \\
3.48^{\mathrm{a}} \\
3.29^{\mathrm{a}}\end{array}$ & $\begin{array}{l}+.55 \\
\pm .56 \\
+.49 \\
\end{array}$ & $\begin{array}{l}\text { Highly Competent } \\
\text { Highly Competent } \\
\text { Highly Competent }\end{array}$ & .180 & $.836^{\mathrm{ns}}$ \\
\hline Supervisor Evaluation & $\begin{array}{c}<2 \text { Prep } \\
\text { 2-4 Preps } \\
>5 \text { Preps } \\
\end{array}$ & $\begin{array}{l}3.96^{\mathrm{a}} \\
3.92^{\mathrm{a}} \\
3.79^{\mathrm{a}} \\
\end{array}$ & $\begin{array}{r}+.55 \\
+.57 \\
+.50 \\
\end{array}$ & $\begin{array}{l}\text { Highly Competent } \\
\text { Highly Competent } \\
\text { Highly Competent }\end{array}$ & .094 & $.911^{\mathrm{ns}}$ \\
\hline \multicolumn{7}{|c|}{ Number of Quasi Assignments } \\
\hline Self-Evaluation & $\begin{array}{l}<2 \text { Quasi } \\
2-4 \text { Quasi } \\
>5 \text { Quasi } \\
\end{array}$ & $\begin{array}{c}3.39^{\mathrm{a}} \\
3.71^{\mathrm{a}} \\
\mathrm{n} / \mathrm{a} \\
\end{array}$ & $\begin{array}{c} \pm .55 \\
\pm .55 \\
\mathrm{n} / \mathrm{a} \\
\end{array}$ & $\begin{array}{l}\text { Highly Competent } \\
\text { Highly Competent } \\
\text { n/a } \\
\end{array}$ & .911 & $.349^{\mathrm{ns}}$ \\
\hline Student Evaluation & $\begin{array}{l}2 \text { Quasi } \\
\text { 2-4 Quasi } \\
>5 \text { Quasi } \\
\end{array}$ & $\begin{array}{c}\mathbf{3 . 1 6}^{\mathbf{b}} \\
3.51^{\mathrm{a}} \\
\mathrm{n} / \mathrm{a} \\
\end{array}$ & $\begin{array}{c}.53 \\
\pm .52 \\
\mathrm{n} / \mathrm{a} \\
\end{array}$ & $\begin{array}{c}\text { Competent } \\
\text { Highly Competent } \\
\text { n/a } \\
\end{array}$ & 5.12 & $.029^{*}$ \\
\hline Supervisor Evaluation & $\begin{array}{l}<2 \text { Quasi } \\
2-4 \text { Quasi } \\
>5 \text { Quasi }\end{array}$ & $\begin{array}{c}3.56^{\mathrm{a}} \\
3.96^{\mathrm{a}} \\
\mathrm{n} / \mathrm{a}\end{array}$ & $\begin{array}{c} \pm .55 \\
\pm .53 \\
\mathrm{n} / \mathrm{a}\end{array}$ & $\begin{array}{l}\text { Highly Competent } \\
\text { Highly Competent } \\
\text { n/a }\end{array}$ & 1.45 & $.239^{\mathrm{ns}}$ \\
\hline
\end{tabular}

Means with different letters are significantly different $(\rho=0.05)$. Values are expressed in mean \pm S.D. Symbols and abbreviations: $* *=$ highly significant; $*$ = significant; $\mathrm{ns}=$ not significant

In Table 4, teaching efficiency as influenced by trainings and workloads is perceived. Teachers with the least number of training points appeared to have the lowest teaching efficiency as depicted by both self-evaluation (3.06+.58) and student evaluation $(2.97 \pm 54)$. Teaching units and number of preparations did not show significant effects on teaching efficiency. However, the number of quasi assignments or the additional facilitator-adviser functions on top of the teaching workload units seemed to influence such competence. As per student evaluation, those teachers with less than two quasi assignments seemed to have lower teaching efficiency $(3.16+.53)$. This is quite interesting since quasi assignments are considered additional workload for the faculty hence should negatively affect performance, the results however appear to oppose this. The result of the study is supported by the study of Rahman et al. [22] where they assessed the training skills of teachers to examine the attitude of the teachers toward teaching and to determine effectiveness of teaching in terms of student achievement. The findings of the study were that the teachers had a positive attitude towards teacher training and its effectiveness in classroom situation including actual instruction and academic work. This indicates that teacher training was positively related to effective teaching. 
Table 5. General class atmosphere as grouped according to different demographic data

\begin{tabular}{|c|c|c|c|c|c|c|}
\hline Type of Evaluation & Variable & Mean & S.D. & Descriptive Equivalent & $\mathrm{F}$ & Sig. \\
\hline \multicolumn{7}{|c|}{ Sex } \\
\hline Self-Evaluation & $\begin{array}{c}\text { Male } \\
\text { Female }\end{array}$ & $\begin{array}{l}3.40^{\mathrm{a}} \\
3.81^{\mathrm{a}}\end{array}$ & $\begin{array}{l} \pm .73 \\
+.59\end{array}$ & $\begin{array}{l}\text { Highly Competent } \\
\text { Highly Competent }\end{array}$ & 1.98 & $.171^{\mathrm{ns}}$ \\
\hline Student Evaluation & $\begin{array}{c}\text { Male } \\
\text { Female }\end{array}$ & $\begin{array}{l}3.22^{b} \\
3.59^{a}\end{array}$ & $\begin{array}{l}+.88 \\
\pm .58 \\
\end{array}$ & $\begin{array}{c}\text { Competent } \\
\text { Highly Competent }\end{array}$ & 1.70 & $.042^{*}$ \\
\hline Supervisor Evaluation & $\begin{array}{c}\text { Male } \\
\text { Female }\end{array}$ & $\begin{array}{l}3.65^{\mathrm{a}} \\
3.91^{\mathrm{a}} \\
\end{array}$ & $\begin{array}{l}+.70 \\
+.57 \\
\end{array}$ & $\begin{array}{l}\text { Highly Competent } \\
\text { Highly Competent }\end{array}$ & 1.85 & $.185^{\mathrm{ns}}$ \\
\hline \multicolumn{7}{|c|}{ Civil Status } \\
\hline Self-Evaluation & $\begin{array}{c}\text { Single } \\
\text { Married }\end{array}$ & $\begin{array}{l}3.67^{\mathrm{a}} \\
3.69^{\mathrm{a}} \\
\end{array}$ & $\begin{array}{r}+.84 \\
+.61 \\
\end{array}$ & $\begin{array}{l}\text { Highly Competent } \\
\text { Highly Competent }\end{array}$ & .007 & $.932^{\text {ns }}$ \\
\hline Student Evaluation & $\begin{array}{c}\text { Single } \\
\text { Married }\end{array}$ & $\begin{array}{l}3.45^{\mathrm{a}} \\
3.48^{\mathrm{a}} \\
\end{array}$ & $\begin{array}{l} \pm .79 \\
\pm .61 \\
\end{array}$ & $\begin{array}{l}\text { Highly Competent } \\
\text { Highly Competent }\end{array}$ & .011 & $.917^{\text {ns }}$ \\
\hline Supervisor Evaluation & $\begin{array}{c}\text { Single } \\
\text { Married }\end{array}$ & $\begin{array}{l}3.85^{\mathrm{a}} \\
3.98^{\mathrm{a}} \\
\end{array}$ & $\begin{array}{l} \pm .79 \\
+.61 \\
\end{array}$ & $\begin{array}{l}\text { Highly Competent } \\
\text { Highly Competent }\end{array}$ & .215 & $.647^{\mathrm{ns}}$ \\
\hline \multicolumn{7}{|c|}{ No. of Children } \\
\hline Self-Evaluation & $\begin{array}{l}\text { None } \\
\text { One } \\
\text { Three }\end{array}$ & $\begin{array}{l}3.67^{\mathrm{a}} \\
3.56^{\mathrm{a}} \\
3.90^{\mathrm{a}} \\
\end{array}$ & $\begin{array}{l}+.84 \\
\pm .60 \\
+.55 \\
\end{array}$ & $\begin{array}{l}\text { Highly Competent } \\
\text { Highly Competent } \\
\text { Highly Competent }\end{array}$ & .500 & $.613^{\mathrm{ns}}$ \\
\hline Student Evaluation & $\begin{array}{l}\text { None } \\
\text { One } \\
\text { Three }\end{array}$ & $\begin{array}{l}3.45^{\mathrm{a}} \\
3.36^{\mathrm{a}} \\
3.85^{\mathrm{a}}\end{array}$ & $\begin{array}{l}+.79 \\
+.60 \\
+.55\end{array}$ & $\begin{array}{l}\text { Highly Competent } \\
\text { Highly Competent } \\
\text { Highly Competent }\end{array}$ & .542 & $.589^{\mathrm{ns}}$ \\
\hline Supervisor Evaluation & $\begin{array}{l}\text { None } \\
\text { One } \\
\text { Three }\end{array}$ & $\begin{array}{l}3.85^{\mathrm{a}} \\
3.86^{\mathrm{a}} \\
3.91^{\mathrm{a}} \\
\end{array}$ & $\begin{array}{l} \pm .79 \\
\pm .60 \\
\pm .70 \\
\end{array}$ & $\begin{array}{l}\text { Highly Competent } \\
\text { Highly Competent } \\
\text { Highly Competent }\end{array}$ & .644 & $.534^{\mathrm{ns}}$ \\
\hline \multicolumn{7}{|c|}{ Highest Educational Attainment } \\
\hline Self-Evaluation & $\begin{array}{c}\text { BS degree } \\
\text { MS units } \\
\text { PhD degree }\end{array}$ & $\begin{array}{l}3.48^{\mathrm{a}} \\
3.69^{\mathrm{a}} \\
3.94^{\mathrm{a}}\end{array}$ & $\begin{array}{l} \pm .51 \\
\pm .99 \\
+.61\end{array}$ & $\begin{array}{l}\text { Highly Competent } \\
\text { Highly Competent } \\
\text { Highly Competent }\end{array}$ & .680 & $.516^{\mathrm{ns}}$ \\
\hline Student Evaluation & $\begin{array}{l}\text { BS degree } \\
\text { MS units } \\
\text { PhD degree }\end{array}$ & $\begin{array}{l}3.29^{\mathrm{a}} \\
3.48^{\mathrm{a}} \\
3.70^{\mathrm{a}}\end{array}$ & $\begin{array}{l}+.51 \\
+.93 \\
+.61\end{array}$ & $\begin{array}{l}\text { Highly Competent } \\
\text { Highly Competent } \\
\text { Highly Competent }\end{array}$ & .598 & $.558^{\mathrm{ns}}$ \\
\hline Supervisor Evaluation & $\begin{array}{c}\text { BS degree } \\
\text { MS units } \\
\text { PhD degree }\end{array}$ & $\begin{array}{l}3.69^{\mathrm{a}} \\
3.91^{\mathrm{a}} \\
3.93^{\mathrm{a}} \\
\end{array}$ & $\begin{array}{r}+.51 \\
+.93 \\
+.61 \\
\end{array}$ & $\begin{array}{l}\text { Highly Competent } \\
\text { Highly Competent } \\
\text { Highly Competent }\end{array}$ & .700 & $.506^{\mathrm{ns}}$ \\
\hline \multicolumn{7}{|c|}{ Length of Service } \\
\hline Self-Evaluation & $\begin{array}{c}<5 \text { years } \\
5-9 \text { years } \\
10-14 \text { years }\end{array}$ & $\begin{array}{l}3.69^{\mathrm{a}} \\
3.98^{\mathrm{a}} \\
3.44^{\mathrm{a}} \\
\end{array}$ & $\begin{array}{l} \pm .79 \\
+.55 \\
\pm .64 \\
\end{array}$ & $\begin{array}{l}\text { Highly Competent } \\
\text { Highly Competent } \\
\text { Highly Competent }\end{array}$ & .710 & $.502^{\mathrm{ns}}$ \\
\hline Student Evaluation & $\begin{array}{c}<5 \text { years } \\
5-9 \text { years } \\
\mathbf{1 0 - 1 4} \text { years }\end{array}$ & $\begin{array}{l}3.48^{\mathrm{a}} \\
3.85^{\mathrm{a}} \\
\mathbf{3 . 2 3}^{\mathrm{a}} \\
\end{array}$ & $\begin{array}{l} \pm .74 \\
+.55 \\
+.64\end{array}$ & $\begin{array}{c}\text { Highly Competent } \\
\text { Highly Competent } \\
\text { Competent }\end{array}$ & .779 & $.470^{\mathrm{ns}}$ \\
\hline Supervisor Evaluation & $\begin{array}{c}<5 \text { years } \\
5-9 \text { years } \\
10-14 \text { years }\end{array}$ & $\begin{array}{l}3.89^{\mathrm{a}} \\
3.98^{\mathrm{a}} \\
3.73^{\mathrm{a}} \\
\end{array}$ & $\begin{array}{l}+.75 \\
+.55 \\
+.70 \\
\end{array}$ & $\begin{array}{l}\text { Highly Competent } \\
\text { Highly Competent } \\
\text { Highly Competent }\end{array}$ & .772 & $.473^{\text {ns }}$ \\
\hline
\end{tabular}

Means with different letters are significantly different $(\rho=0.05)$. Values are expressed in mean \pm S.D. Symbols and abbreviations: $* *=$ highly significant; * = significant; $\mathrm{ns}=$ not significant

The general class atmosphere as grouped according to demographic data is presented in Table 5. As shown in the table, male teachers appear to be less competent than female teachers when it comes to having a more conducive learning environment. Though gender may appear to affect competence, attaining a conducive learning environment involves several interconnecting factors including the physical atmosphere, the teacher, the learner, the teaching and learning process and other extraneous factors. Civil status, number of children and highest educational attainment appear to have no substantial impact on class atmosphere because all respondents are highly competent regardless of how such variables were grouped. In a different demographic perspective, it appears that the length of service affects general class atmosphere since those respondents with the longest length of teaching service have a less conducive learning atmosphere $(3.23 \pm 64)$ than those with less teaching experience.

No substantial body of literature has indicated that female teachers are more competent than male teachers in terms of general class atmosphere. Other published data would even contradict this finding such as the study of Wenglinsky[23] where he pointed out that more male teachers have innate ability to get students' attention through funny motivations thus having a lighter class atmosphere. On the other hand, Wayne and Young[24] mentioned that there are more female education students than male, and at the same time, there are also more female teachers in the school of education. As a result, female students often tend to get more comfortable dealing and 
working with female teachers than male. Looking into another parameter, the length of service, it seems that teachers with longer length of service have lower competence in terms of having a more conducive learning environment (general class atmosphere). This result is quite interesting because previous studies by Bill[25], Delaney[26], and Stronge[27] have indicated that the longer a teacher has been on his service, the more techniques and pedagogies he possesses which he could use to improve the atmosphere and learning inside the classroom. However, it is possible that teachers having been in the academe for longer periods may also have more rigid disciplinary measures which may consequently affect conduciveness of learning [28].

The general class atmosphere as affected by trainings and workloads is depicted in Table 6 . This table provides sufficient evidence that low number of trainings points would lead to low competence when it comes to manifesting pleasant teaching atmosphere and maintaining discipline among students. As per self-evaluation, those teachers with less than 10 training points are only fairly competent (2.31 \pm .55$)$. Similarly, as per student evaluation, those teachers with less than 10 points are also fairly competent $(2.22+.55)$. And, for supervisor evaluation, those science instructors with less than 10 training points are less competent $(2.62 \pm 53)$ than those with greater number of trainings. For the other parameters, teaching units, number of preparations and number of quasi assignments, it appeared that they have no significant differences among the rest of the variables. Based on the results of the study, trainings definitely equip teachers to have better grasp of what 'conducive learning environment' is [29]. In a study on the impact of teachers' qualification on students' performance, Colfalter et al.[30] found that a significant different exists in the mean competence of teachers with more trainings than those with fewer trainings when it comes to its influence on their teaching environment and performance.

Table 6. General class atmosphere as affected by trainings and workloads

\begin{tabular}{|c|c|c|c|c|c|c|}
\hline Type of Evaluation & Variable & Mean & S.D. & Descriptive Equivalent & $\mathrm{F}$ & Sig. \\
\hline \multicolumn{7}{|c|}{ Training Points } \\
\hline Self-Evaluation & $\begin{array}{l}<\mathbf{1 0} \text { pts. } \\
11-20 \text { pts. } \\
>20 \text { pts. }\end{array}$ & $\begin{array}{l}\mathbf{2 . 3 1} 1^{\mathrm{b}} \\
3.81^{\mathrm{a}} \\
3.96^{\mathrm{a}}\end{array}$ & $\begin{array}{l}+.55 \\
\pm .51 \\
+.55\end{array}$ & $\begin{array}{l}\text { Fairly Competent } \\
\text { Highly Competent } \\
\text { Highly Competent }\end{array}$ & 4.15 & $.006^{* *}$ \\
\hline Student Evaluation & $\begin{array}{l}<\mathbf{1 0} \text { pts. } \\
11-20 \text { pts. } \\
>20 \text { pts. }\end{array}$ & $\begin{array}{l}\mathbf{2 . 2 2 ^ { \mathrm { b } }} \\
3.60^{\mathrm{a}} \\
3.81^{\mathrm{a}}\end{array}$ & $\begin{array}{l} \pm .55 \\
\pm .54 \\
\pm .54\end{array}$ & $\begin{array}{l}\text { Fairly Competent } \\
\text { Highly Competent } \\
\text { Highly Competent }\end{array}$ & 3.59 & $.012^{*}$ \\
\hline Supervisor Evaluation & $\begin{array}{c}<\mathbf{1 0} \text { pts. } \\
11-20 \text { pts. } \\
>20 \text { pts. }\end{array}$ & $\begin{array}{l}\mathbf{2 . 6 2 ^ { \mathrm { b } }} \\
3.85^{\mathrm{a}} \\
3.99^{\mathrm{a}} \\
\end{array}$ & $\begin{array}{l} \pm .53 \\
\pm .57 \\
+.55 \\
\end{array}$ & $\begin{array}{c}\text { Competent } \\
\text { Highly Competent } \\
\text { Highly Competent }\end{array}$ & 3.64 & $.012^{*}$ \\
\hline \multicolumn{7}{|c|}{ Teaching Units } \\
\hline Self-Evaluation & $\begin{array}{l}<20 \text { units } \\
>21 \text { units }\end{array}$ & $\begin{array}{l}3.76^{\mathrm{a}} \\
3.56^{\mathrm{a}} \\
\end{array}$ & $\begin{array}{l} \pm .60 \\
\pm .89 \\
\end{array}$ & $\begin{array}{l}\text { Highly Competent } \\
\text { Highly Competent }\end{array}$ & .479 & $.495^{\text {ns }}$ \\
\hline Student Evaluation & $\begin{array}{l}<20 \text { units } \\
>21 \text { units }\end{array}$ & $\begin{array}{l}3.55^{\mathrm{a}} \\
3.36^{\mathrm{a}} \\
\end{array}$ & $\begin{array}{l} \pm .58 \\
\pm .73 \\
\end{array}$ & $\begin{array}{l}\text { Highly Competent } \\
\text { Highly Competent }\end{array}$ & .455 & $.506^{\mathrm{ns}}$ \\
\hline Supervisor Evaluation & $\begin{array}{l}<20 \text { units } \\
>21 \text { units }\end{array}$ & $\begin{array}{l}3.94^{\mathrm{a}} \\
3.76^{\mathrm{a}} \\
\end{array}$ & $\begin{array}{l}+.59 \\
\pm .83 \\
\end{array}$ & $\begin{array}{l}\text { Highly Competent } \\
\text { Highly Competent }\end{array}$ & .938 & $.342^{\text {ns }}$ \\
\hline \multicolumn{7}{|c|}{ Number of Preparations } \\
\hline Self-Evaluation & $\begin{array}{l}<2 \text { Prep } \\
\text { 2-4 Preps } \\
>5 \text { Preps }\end{array}$ & $\begin{array}{l}3.94^{\mathrm{a}} \\
3.90^{\mathrm{a}} \\
3.52^{\mathrm{a}}\end{array}$ & $\begin{array}{l} \pm .55 \\
\pm .54 \\
\pm .80\end{array}$ & $\begin{array}{l}\text { Highly Competent } \\
\text { Highly Competent } \\
\text { Highly Competent }\end{array}$ & 1.20 & $.318^{\mathrm{ns}}$ \\
\hline Student Evaluation & $\begin{array}{l}<2 \text { Prep } \\
\text { 2-4 Preps } \\
>5 \text { Preps }\end{array}$ & $\begin{array}{l}3.85^{\mathrm{a}} \\
3.72^{\mathrm{a}} \\
3.31^{\mathrm{a}}\end{array}$ & $\begin{array}{l}+.53 \\
+.76 \\
+.50\end{array}$ & $\begin{array}{l}\text { Highly Competent } \\
\text { Highly Competent } \\
\text { Highly Competent }\end{array}$ & 1.27 & $.299^{\mathrm{ns}}$ \\
\hline Supervisor Evaluation & $\begin{array}{c}2 \text { Prep } \\
2-4 \text { Preps } \\
>5 \text { Preps } \\
\end{array}$ & $\begin{array}{l}3.95^{\mathrm{a}} \\
3.90^{\mathrm{a}} \\
3.75^{\mathrm{a}} \\
\end{array}$ & $\begin{array}{l}+.55 \\
\pm .75 \\
\pm .49 \\
\end{array}$ & $\begin{array}{l}\text { Highly Competent } \\
\text { Highly Competent } \\
\text { Highly Competent }\end{array}$ & 1.51 & $.242^{\text {ns }}$ \\
\hline \multicolumn{7}{|c|}{ Number of Quasi Assignments } \\
\hline Self-Evaluation & $\begin{array}{l}<2 \text { Quasi } \\
2-4 \text { Quasi } \\
>5 \text { Quasi } \\
\end{array}$ & $\begin{array}{c}3.81^{\mathrm{a}} \\
3.66^{\mathrm{a}} \\
\mathrm{n} / \mathrm{a} \\
\end{array}$ & $\begin{array}{r} \pm .55 \\
\pm .76 \\
\mathrm{n} / \mathrm{a} \\
\end{array}$ & $\begin{array}{c}\text { Highly Competent } \\
\text { Highly Competent } \\
\text { n/a }\end{array}$ & .115 & $.737^{\mathrm{ns}}$ \\
\hline Student Evaluation & $\begin{array}{l}<2 \text { Quasi } \\
2-4 \text { Quasi } \\
>5 \text { Quasi }\end{array}$ & $\begin{array}{c}3.58^{\mathrm{a}} \\
3.45^{\mathrm{a}} \\
\mathrm{n} / \mathrm{a}\end{array}$ & $\begin{array}{c} \pm .55 \\
\pm .73 \\
\mathrm{n} / \mathrm{a}\end{array}$ & $\begin{array}{l}\text { Highly Competent } \\
\text { Highly Competent } \\
\text { n/a }\end{array}$ & .092 & $.764^{\mathrm{ns}}$ \\
\hline Supervisor Evaluation & $\begin{array}{l}<2 \text { Quasi } \\
2-4 \text { Quasi } \\
>5 \text { Quasi }\end{array}$ & $\begin{array}{c}3.98^{\mathrm{a}} \\
3.90^{\mathrm{a}} \\
\mathrm{n} / \mathrm{a} \\
\end{array}$ & $\begin{array}{c} \pm .55 \\
\pm .73 \\
\mathrm{n} / \mathrm{a}\end{array}$ & $\begin{array}{c}\text { Highly Competent } \\
\text { Highly Competent } \\
\text { n/a }\end{array}$ & .036 & $.852^{\text {ns }}$ \\
\hline
\end{tabular}

Means with different letters are significantly different $(\rho=0.05)$. Values are expressed in mean \pm S.D. Symbols and abbreviations: $* *=$ highly significant; $*$ = significant; $\mathrm{ns}=$ not significant 
Table 7. Substantiality in teaching as grouped according to different demographic data

\begin{tabular}{|c|c|c|c|c|c|c|}
\hline Type of Evaluation & Variable & Mean & S.D. & Descriptive Equivalent & $\mathrm{F}$ & Sig. \\
\hline \multicolumn{7}{|c|}{ Sex } \\
\hline Self-Evaluation & $\begin{array}{c}\text { Male } \\
\text { Female }\end{array}$ & $\begin{array}{l}3.53^{\mathrm{a}} \\
3.86^{\mathrm{a}}\end{array}$ & $\begin{array}{l} \pm .71 \\
+.50\end{array}$ & $\begin{array}{l}\text { Highly Competent } \\
\text { Highly Competent }\end{array}$ & 1.97 & $.173^{\mathrm{ns}}$ \\
\hline Student Evaluation & $\begin{array}{c}\text { Male } \\
\text { Female }\end{array}$ & $\begin{array}{l}3.35^{\mathrm{a}} \\
3.64^{\mathrm{a}} \\
\end{array}$ & $\begin{array}{l}+.66 \\
+.51 \\
\end{array}$ & $\begin{array}{l}\text { Highly Competent } \\
\text { Highly Competent }\end{array}$ & 1.58 & $.219^{\mathrm{n}}$ \\
\hline Supervisor Evaluation & $\begin{array}{c}\text { Male } \\
\text { Female }\end{array}$ & $\begin{array}{l}3.78^{\mathrm{a}} \\
3.90^{\mathrm{a}}\end{array}$ & $\begin{array}{l}+.67 \\
+.57 \\
\end{array}$ & $\begin{array}{l}\text { Highly Competent } \\
\text { Highly Competent }\end{array}$ & 1.76 & $.196^{\mathrm{ns}}$ \\
\hline \multicolumn{7}{|c|}{ Civil Status } \\
\hline Self-Evaluation & $\begin{array}{c}\text { Single } \\
\text { Married }\end{array}$ & $\begin{array}{l}3.74^{\mathrm{a}} \\
3.76^{\mathrm{a}}\end{array}$ & $\begin{array}{l}+.67 \\
+.50\end{array}$ & $\begin{array}{l}\text { Highly Competent } \\
\text { Highly Competent }\end{array}$ & .007 & $.933^{\mathrm{n}}$ \\
\hline Student Evaluation & $\begin{array}{c}\text { Single } \\
\text { Married }\end{array}$ & $\begin{array}{l}3.53^{\mathrm{a}} \\
3.55^{\mathrm{a}}\end{array}$ & $\begin{array}{l}+.62 \\
+.50 \\
\end{array}$ & $\begin{array}{l}\text { Highly Competent } \\
\text { Highly Competent }\end{array}$ & .012 & $.914^{\mathrm{ns}}$ \\
\hline Supervisor Evaluation & $\begin{array}{c}\text { Single } \\
\text { Married }\end{array}$ & $\begin{array}{l}3.93^{\mathrm{a}} \\
3.99^{\mathrm{a}} \\
\end{array}$ & $\begin{array}{l} \pm .62 \\
+.51 \\
\end{array}$ & $\begin{array}{l}\text { Highly Competent } \\
\text { Highly Competent }\end{array}$ & .310 & $.583^{\mathrm{ns}}$ \\
\hline \multicolumn{7}{|c|}{ No. of Children } \\
\hline Self-Evaluation & $\begin{array}{l}\text { None } \\
\text { One } \\
\text { Three }\end{array}$ & $\begin{array}{l}3.74^{\mathrm{a}} \\
3.80^{\mathrm{a}} \\
3.67^{\mathrm{a}} \\
\end{array}$ & $\begin{array}{l}+.67 \\
+.55 \\
+.59 \\
\end{array}$ & $\begin{array}{l}\text { Highly Competent } \\
\text { Highly Competent } \\
\text { Highly Competent }\end{array}$ & .056 & $.945^{\mathrm{ns}}$ \\
\hline Student Evaluation & $\begin{array}{l}\text { None } \\
\text { One } \\
\text { Three }\end{array}$ & $\begin{array}{l}3.53^{\mathrm{a}} \\
3.59^{\mathrm{a}} \\
3.45^{\mathrm{a}} \\
\end{array}$ & $\begin{array}{l} \pm .63 \\
\pm .51 \\
+.56\end{array}$ & $\begin{array}{l}\text { Highly Competent } \\
\text { Highly Competent } \\
\text { Highly Competent }\end{array}$ & .067 & $.936^{\mathrm{n}}$ \\
\hline Supervisor Evaluation & $\begin{array}{l}\text { None } \\
\text { One } \\
\text { Three }\end{array}$ & $\begin{array}{l}3.93^{\mathrm{a}} \\
3.99^{\mathrm{a}} \\
3.96^{\mathrm{a}} \\
\end{array}$ & $\begin{array}{l} \pm .62 \\
+.51 \\
+.57 \\
\end{array}$ & $\begin{array}{l}\text { Highly Competent } \\
\text { Highly Competent } \\
\text { Highly Competent }\end{array}$ & .210 & $.812^{\mathrm{ns}}$ \\
\hline \multicolumn{7}{|c|}{ Highest Educational Attainment } \\
\hline Self-Evaluation & $\begin{array}{c}\text { BS degree } \\
\text { MS units } \\
\text { PhD degree }\end{array}$ & $\begin{array}{l}3.66^{\mathrm{a}} \\
3.76^{\mathrm{a}} \\
3.86^{\mathrm{a}} \\
\end{array}$ & $\begin{array}{l} \pm .54 \\
\pm .76 \\
+.50 \\
\end{array}$ & $\begin{array}{l}\text { Highly Competent } \\
\text { Highly Competent } \\
\text { Highly Competent }\end{array}$ & .196 & $.823^{\mathrm{n}}$ \\
\hline Student Evaluation & $\begin{array}{c}\text { BS degree } \\
\text { MS units } \\
\text { PhD degree }\end{array}$ & $\begin{array}{l}3.47^{\mathrm{a}} \\
3.55^{\mathrm{a}} \\
3.62^{\mathrm{a}} \\
\end{array}$ & $\begin{array}{l}+.53 \\
+.71 \\
+.56 \\
\end{array}$ & $\begin{array}{l}\text { Highly Competent } \\
\text { Highly Competent } \\
\text { Highly Competent }\end{array}$ & .129 & $.880^{\mathrm{n}}$ \\
\hline Supervisor Evaluation & $\begin{array}{c}\text { BS degree } \\
\text { MS units } \\
\text { PhD degree }\end{array}$ & $\begin{array}{l}3.87^{\mathrm{a}} \\
3.92^{\mathrm{a}} \\
3.99^{\mathrm{a}} \\
\end{array}$ & $\begin{array}{l}+.53 \\
+.71 \\
+.57 \\
\end{array}$ & $\begin{array}{l}\text { Highly Competent } \\
\text { Highly Competent } \\
\text { Highly Competent }\end{array}$ & .273 & $.763^{\mathrm{ns}}$ \\
\hline \multicolumn{7}{|c|}{ Length of Service } \\
\hline Self-Evaluation & $\begin{array}{c}<5 \text { years } \\
5-9 \text { years } \\
10-14 \text { years }\end{array}$ & $\begin{array}{l}3.66^{\mathrm{a}} \\
3.73^{\mathrm{a}} \\
3.86^{\mathrm{a}}\end{array}$ & $\begin{array}{l} \pm .63 \\
+.54 \\
\pm .59\end{array}$ & $\begin{array}{l}\text { Highly Competent } \\
\text { Highly Competent } \\
\text { Highly Competent }\end{array}$ & .142 & $.868^{\mathrm{ns}}$ \\
\hline Student Evaluation & $\begin{array}{c}<5 \text { years } \\
5-9 \text { years } \\
10-14 \text { years }\end{array}$ & $\begin{array}{l}3.45^{\mathrm{a}} \\
3.52^{\mathrm{a}} \\
3.65^{\mathrm{a}}\end{array}$ & $\begin{array}{l}+.60 \\
+.55 \\
+.54\end{array}$ & $\begin{array}{l}\text { Highly Competent } \\
\text { Highly Competent } \\
\text { Highly Competent }\end{array}$ & .155 & $.857^{\mathrm{ns}}$ \\
\hline Supervisor Evaluation & $\begin{array}{c}<5 \text { years } \\
5-9 \text { years } \\
10-14 \text { years }\end{array}$ & $\begin{array}{l}3.94^{\mathrm{a}} \\
3.95^{\mathrm{a}} \\
3.99^{\mathrm{a}}\end{array}$ & $\begin{array}{l}+.61 \\
+.54 \\
\pm .57\end{array}$ & $\begin{array}{l}\text { Highly Competent } \\
\text { Highly Competent } \\
\text { Highly Competent }\end{array}$ & .313 & $.734^{\mathrm{ns}}$ \\
\hline
\end{tabular}

Means with different letters are significantly different $(\rho=0.05)$. Values are expressed in mean \pm S.D. Symbols and abbreviations: $* *=$ highly significant; * = significant; $\mathrm{ns}=$ not significant

Table 7 recognizes how substantiality in teaching is influenced by science teachers' demographic data. Although all parameters appear to be not statistically significant, there are a few points that can be highlighted. Female respondents appear to have an edge when it comes to the substance of what they are teaching. This may imply that female teachers tend to be more comfortable in presenting and discussing their subject content in a female-dominated audience. Married respondents were also found to have higher mastery in terms of teaching substantiality. This may suggest that married respondents are less conscious and more elaborate in conversing and expounding their subject to teach. Lastly, it can also be observed in the table above that substantiality in teaching is increased by higher educational attainment and length of service. On the other hand, Table 8 presents the substantiality in teaching and how it is affected by both trainings and workloads. It can be gleaned from the table that teachers with the least substantiality in teaching are those with the least number of training points. (self-evaluation $=2.86 \pm .55$; student evaluation $=2.77 \pm .53$; and supervisor evaluation $=3.17 \pm .43$ ). 
Table 8. Substantiality in teaching as affected by trainings and workloads

\begin{tabular}{|c|c|c|c|c|c|c|}
\hline Type of Evaluation & Variable & Mean & S.D. & Descriptive Equivalent & $\mathrm{F}$ & Sig. \\
\hline \multicolumn{7}{|c|}{ Training Points } \\
\hline Self-Evaluation & $\begin{array}{l}<\mathbf{1 0} \text { pts. } \\
11-20 \text { pts. } \\
>20 \text { pts. }\end{array}$ & $\begin{array}{l}\mathbf{2 . 8 6} \mathbf{b}^{\mathrm{b}} \\
3.86^{\mathrm{a}} \\
3.98^{\mathrm{a}}\end{array}$ & $\begin{array}{l} \pm .55 \\
\pm .54 \\
+.55\end{array}$ & $\begin{array}{c}\text { Competent } \\
\text { Highly Competent } \\
\text { Highly Competent }\end{array}$ & 3.60 & $.019^{*}$ \\
\hline Student Evaluation & $\begin{array}{l}<\mathbf{1 0} \text { pts. } \\
11-20 \text { pts. } \\
>20 \text { pts. }\end{array}$ & 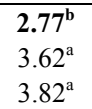 & $\begin{array}{l} \pm .53 \\
\pm .56 \\
+.52\end{array}$ & $\begin{array}{c}\text { Competent } \\
\text { Highly Competent } \\
\text { Highly Competent }\end{array}$ & 3.24 & $0.032^{*}$ \\
\hline Supervisor Evaluation & $\begin{array}{l}<\mathbf{1 0} \text { pts. } \\
11-20 \text { pts. } \\
>20 \text { pts. }\end{array}$ & $\begin{array}{l}\mathbf{3 . 1 7 ^ { \mathbf { b } }} \\
3.89^{\mathrm{a}} \\
3.97^{\mathrm{a}} \\
\end{array}$ & $\begin{array}{l}+.43 \\
\pm .44 \\
+.59 \\
\end{array}$ & $\begin{array}{c}\text { Competent } \\
\text { Highly Competent } \\
\text { Highly Competent } \\
\end{array}$ & 3.29 & $0.030^{*}$ \\
\hline \multicolumn{7}{|c|}{ Teaching Units } \\
\hline Self-Evaluation & $\begin{array}{l}<20 \text { units } \\
>21 \text { units }\end{array}$ & $\begin{array}{l}3.82^{\mathrm{a}} \\
3.67^{\mathrm{a}} \\
\end{array}$ & $\begin{array}{l} \pm .51 \\
\pm .69 \\
\end{array}$ & $\begin{array}{l}\text { Highly Competent } \\
\text { Highly Competent }\end{array}$ & .476 & $.497^{\mathrm{ns}}$ \\
\hline Student Evaluation & $\begin{array}{l}<20 \text { units } \\
>21 \text { units }\end{array}$ & $\begin{array}{l}3.61^{\mathrm{a}} \\
3.46^{\mathrm{a}} \\
\end{array}$ & $\begin{array}{l} \pm .50 \\
\pm .64 \\
\end{array}$ & $\begin{array}{l}\text { Highly Competent } \\
\text { Highly Competent }\end{array}$ & .436 & $.515^{\mathrm{ns}}$ \\
\hline Supervisor Evaluation & $\begin{array}{l}<20 \text { units } \\
>21 \text { units }\end{array}$ & $\begin{array}{l}3.98^{\mathrm{a}} \\
3.86^{\mathrm{a}} \\
\end{array}$ & $\begin{array}{l} \pm .49 \\
\pm .67 \\
\end{array}$ & $\begin{array}{l}\text { Highly Competent } \\
\text { Highly Competent }\end{array}$ & 1.05 & $.314^{\mathrm{ns}}$ \\
\hline \multicolumn{7}{|c|}{ Number of Preparations } \\
\hline Self-Evaluation & $\begin{array}{c}2 \text { Prep } \\
2-4 \text { Preps } \\
>5 \text { Preps } \\
\end{array}$ & $\begin{array}{l}3.86^{\mathrm{a}} \\
3.73^{\mathrm{a}} \\
3.67^{\mathrm{a}} \\
\end{array}$ & $\begin{array}{l} \pm .55 \\
\pm .63 \\
+.59 \\
\end{array}$ & $\begin{array}{l}\text { Highly Competent } \\
\text { Highly Competent } \\
\text { Highly Competent }\end{array}$ & .142 & $.868^{\mathrm{ns}}$ \\
\hline Student Evaluation & $\begin{array}{l}<2 \text { Prep } \\
2-4 \text { Preps } \\
>5 \text { Preps }\end{array}$ & $\begin{array}{l}3.64^{\mathrm{a}} \\
3.52^{\mathrm{a}} \\
3.45^{\mathrm{a}}\end{array}$ & $\begin{array}{l} \pm .43 \\
\pm .60 \\
+.56\end{array}$ & $\begin{array}{l}\text { Highly Competent } \\
\text { Highly Competent } \\
\text { Highly Competent }\end{array}$ & .133 & $.876^{\mathrm{ns}}$ \\
\hline Supervisor Evaluation & $\begin{array}{c}<2 \text { Prep } \\
\text { 2-4 Preps } \\
>5 \text { Preps } \\
\end{array}$ & $\begin{array}{l}3.96^{\mathrm{a}} \\
3.90^{\mathrm{a}} \\
3.85^{\mathrm{a}} \\
\end{array}$ & $\begin{array}{l}.55 \\
\pm .61 \\
+.57 \\
\end{array}$ & $\begin{array}{l}\text { Highly Competent } \\
\text { Highly Competent } \\
\text { Highly Competent }\end{array}$ & .126 & $.882^{\mathrm{ns}}$ \\
\hline \multicolumn{7}{|c|}{ Number of Quasi Assignments } \\
\hline Self-Evaluation & $\begin{array}{l}<2 \text { Quasi } \\
2-4 \text { Quasi } \\
>5 \text { Quasi } \\
\end{array}$ & $\begin{array}{c}3.66^{\mathrm{a}} \\
3.76^{\mathrm{a}} \\
\mathrm{n} / \mathrm{a} \\
\end{array}$ & $\begin{array}{c} \pm .46 \\
\pm .43 \\
\mathrm{n} / \mathrm{a} \\
\end{array}$ & $\begin{array}{c}\text { Highly Competent } \\
\text { Highly Competent } \\
\text { n/a } \\
\end{array}$ & .073 & $.789^{\mathrm{ns}}$ \\
\hline Student Evaluation & $\begin{array}{l}<2 \text { Quasi } \\
2-4 \text { Quasi } \\
>5 \text { Quasi } \\
\end{array}$ & $\begin{array}{c}3.43^{\mathrm{a}} \\
3.55^{\mathrm{a}} \\
\mathrm{n} / \mathrm{a} \\
\end{array}$ & $\begin{array}{c} \pm .47 \\
\pm .58 \\
\mathrm{n} / \mathrm{a} \\
\end{array}$ & $\begin{array}{c}\text { Highly Competent } \\
\text { Highly Competent } \\
\text { n/a } \\
\end{array}$ & .120 & $.731^{\mathrm{ns}}$ \\
\hline Supervisor Evaluation & $\begin{array}{l}<2 \text { Quasi } \\
2-4 \text { Quasi } \\
>5 \text { Quasi }\end{array}$ & $\begin{array}{c}3.83^{\mathrm{a}} \\
3.99^{\mathrm{a}} \\
\mathrm{n} / \mathrm{a}\end{array}$ & $\begin{array}{c} \pm .46 \\
\pm .40 \\
\mathrm{n} / \mathrm{a} \\
\end{array}$ & $\begin{array}{c}\text { Highly Competent } \\
\text { Highly Competent } \\
\text { n/a }\end{array}$ & .237 & $.631^{\mathrm{ns}}$ \\
\hline
\end{tabular}

Means with different letters are significantly different $(\rho=0.05)$. Values are expressed in mean \pm S.D. Symbols and abbreviations: $* *=$ highly significant; $*$ = significant; $\mathrm{ns}=$ not significant

This shows how important teacher trainings and seminars are to increase the knowledge of teachers and their mastery to the subject matter. On the other hand, teaching unit and number of quasi assignments do not have any statistically significant differences among the different variables being set. For the number of various course subjects to teach (preparations), it appears that an increased number of preparations reduce the substantiality in teaching. Similar to the results of this study, Ukewe[31] also observed that educational training influences job performance and also acts as a reliable indicator of the substance of information that the teacher is relaying.

Table 9 below shows how evaluation skills may be influenced by demographic data. As shown, male teachers appear to have slightly less competence in their evaluation skills. This can be observed for all the three evaluators: self-evaluation: $3.24 \pm 64$, student evaluation: $3.06 \pm 60$, and supervisor evaluation $3.49 \pm 61$. Sex may therefore have an impact on teacher's evaluation skills. Civil status and number of children do not have significant influences on the evaluation skills of teachers. However, the degree and units earned influences teachers' evaluation skills. 
Table 9. Evaluation skills as grouped according to different demographic data

\begin{tabular}{|c|c|c|c|c|c|c|}
\hline Type of Evaluation & Variable & Mean & S.D. & Descriptive Equivalent & $\mathrm{F}$ & Sig. \\
\hline \multicolumn{7}{|c|}{ Sex } \\
\hline Self-Evaluation & $\begin{array}{c}\text { Male } \\
\text { Female }\end{array}$ & $\begin{array}{l}3.24^{\mathrm{b}} \\
3.75^{\mathrm{a}} \\
\end{array}$ & $\begin{array}{l} \pm .64 \\
\pm .48 \\
\end{array}$ & $\begin{array}{c}\text { Competent } \\
\text { Highly Competent } \\
\end{array}$ & 5.36 & $.029^{*}$ \\
\hline Student Evaluation & $\begin{array}{l}\text { Male } \\
\text { Female }\end{array}$ & $\begin{array}{l}\mathbf{3 . 0 6}^{\mathbf{b}} \\
3.53^{\mathrm{a}}\end{array}$ & $\begin{array}{l}+.60 \\
+.48\end{array}$ & $\begin{array}{c}\text { Competent } \\
\text { Highly Competent }\end{array}$ & 4.77 & $.038^{*}$ \\
\hline Supervisor Evaluation & $\begin{array}{c}\text { Male } \\
\text { Female }\end{array}$ & $\begin{array}{l}3.49^{\mathbf{b}} \\
3.98^{\mathrm{a}} \\
\end{array}$ & $\begin{array}{l}+.61 \\
+.56 \\
\end{array}$ & $\begin{array}{c}\text { Highly Competent } \\
\text { Highly Competent }\end{array}$ & 5.10 & $.033^{*}$ \\
\hline \multicolumn{7}{|c|}{ Civil Status } \\
\hline Self-Evaluation & $\begin{array}{l}\text { Single } \\
\text { Married }\end{array}$ & $\begin{array}{l}3.56^{\mathrm{a}} \\
3.61^{\mathrm{a}} \\
\end{array}$ & $\begin{array}{l} \pm .66 \\
+.51 \\
\end{array}$ & $\begin{array}{l}\text { Highly Competent } \\
\text { Highly Competent }\end{array}$ & .027 & $.871^{\mathrm{ns}}$ \\
\hline Student Evaluation & $\begin{array}{l}\text { Single } \\
\text { Married }\end{array}$ & $\begin{array}{l}3.35^{\mathrm{a}} \\
3.39^{\mathrm{a}} \\
\end{array}$ & $\begin{array}{l} \pm .62 \\
+.50 \\
\end{array}$ & $\begin{array}{l}\text { Highly Competent } \\
\text { Highly Competent }\end{array}$ & .037 & $.850^{\mathrm{ns}}$ \\
\hline Supervisor Evaluation & $\begin{array}{l}\text { Single } \\
\text { Married }\end{array}$ & $\begin{array}{l}3.75^{\mathrm{a}} \\
3.89^{\mathrm{a}} \\
\end{array}$ & $\begin{array}{l} \pm .62 \\
+.59 \\
\end{array}$ & $\begin{array}{l}\text { Highly Competent } \\
\text { Highly Competent }\end{array}$ & .413 & $.526^{\mathrm{ns}}$ \\
\hline \multicolumn{7}{|c|}{ No. of Children } \\
\hline Self-Evaluation & $\begin{array}{l}\text { None } \\
\text { One } \\
\text { Three }\end{array}$ & $\begin{array}{l}3.56^{\mathrm{a}} \\
3.54^{\mathrm{a}} \\
3.78^{\mathrm{a}} \\
\end{array}$ & $\begin{array}{l}+.66 \\
\pm .51 \\
+.55 \\
\end{array}$ & $\begin{array}{l}\text { Highly Competent } \\
\text { Highly Competent } \\
\text { Highly Competent } \\
\end{array}$ & .190 & $.828^{\mathrm{ns}}$ \\
\hline Student Evaluation & $\begin{array}{l}\text { None } \\
\text { One } \\
\text { Three }\end{array}$ & $\begin{array}{l}3.35^{\mathrm{a}} \\
3.34^{\mathrm{a}} \\
3.57^{\mathrm{a}}\end{array}$ & $\begin{array}{l} \pm .62 \\
\pm .54 \\
+.53 \\
\end{array}$ & $\begin{array}{l}\text { Highly Competent } \\
\text { Highly Competent } \\
\text { Highly Competent }\end{array}$ & .205 & $.816^{\mathrm{ns}}$ \\
\hline Supervisor Evaluation & $\begin{array}{l}\text { None } \\
\text { One } \\
\text { Three } \\
\end{array}$ & $\begin{array}{l}3.75^{\mathrm{a}} \\
3.84^{\mathrm{a}} \\
3.93^{\mathrm{a}} \\
\end{array}$ & $\begin{array}{l}+.62 \\
\pm .50 \\
+.53 \\
\end{array}$ & $\begin{array}{l}\text { Highly Competent } \\
\text { Highly Competent } \\
\text { Highly Competent } \\
\end{array}$ & .389 & $.682^{\text {ns }}$ \\
\hline \multicolumn{7}{|c|}{ Highest Educational Attainment } \\
\hline Self-Evaluation & $\begin{array}{l}\text { BS degree } \\
\text { MS units } \\
\text { PhD degree }\end{array}$ & $\begin{array}{l}3.39^{\mathrm{a}} \\
3.60^{\mathrm{a}} \\
3.83^{\mathrm{a}}\end{array}$ & $\begin{array}{l} \pm .51 \\
\pm .71 \\
+.50\end{array}$ & $\begin{array}{l}\text { Highly Competent } \\
\text { Highly Competent } \\
\text { Highly Competent }\end{array}$ & 1.02 & $.376^{\mathrm{ns}}$ \\
\hline Student Evaluation & $\begin{array}{c}\text { BS degree } \\
\text { MS units } \\
\text { PhD degree }\end{array}$ & $\begin{array}{l}3.20^{\mathrm{a}} \\
3.59^{\mathrm{a}} \\
3.60^{\mathrm{a}} \\
\end{array}$ & $\begin{array}{l} \pm .66 \\
\pm .52 \\
+.51 \\
\end{array}$ & $\begin{array}{c}\text { Competent } \\
\text { Highly Competent } \\
\text { Highly Competent } \\
\end{array}$ & .886 & $.425^{\mathrm{ns}}$ \\
\hline Supervisor Evaluation & $\begin{array}{l}\text { BS degree } \\
\text { MS units } \\
\text { PhD degree }\end{array}$ & $\begin{array}{l}3.60^{\mathrm{a}} \\
3.60^{\mathrm{a}} \\
3.89^{\mathrm{a}}\end{array}$ & $\begin{array}{l} \pm .52 \\
\pm .63 \\
+.59 \\
\end{array}$ & $\begin{array}{l}\text { Highly Competent } \\
\text { Highly Competent } \\
\text { Highly Competent }\end{array}$ & .108 & $.356^{\mathrm{ns}}$ \\
\hline \multicolumn{7}{|c|}{ Length of Service } \\
\hline Self-Evaluation & $\begin{array}{c}5 \text { years } \\
5-9 \text { years } \\
10-14 \text { years } \\
\end{array}$ & $\begin{array}{l}3.53^{\mathrm{a}} \\
3.78^{\mathrm{a}} \\
3.65^{\mathrm{a}} \\
\end{array}$ & $\begin{array}{l} \pm .63 \\
\pm .55 \\
\pm .49 \\
\end{array}$ & $\begin{array}{l}\text { Highly Competent } \\
\text { Highly Competent } \\
\text { Highly Competent } \\
\end{array}$ & .279 & $.759^{\mathrm{ns}}$ \\
\hline Student Evaluation & $\begin{array}{c}<5 \text { years } \\
5-9 \text { years } \\
10-14 \text { years }\end{array}$ & $\begin{array}{l}3.32^{\mathrm{a}} \\
3.57^{\mathrm{a}} \\
3.44^{\mathrm{a}}\end{array}$ & $\begin{array}{l}+.60 \\
+.55 \\
+.48 \\
\end{array}$ & $\begin{array}{l}\text { Highly Competent } \\
\text { Highly Competent } \\
\text { Highly Competent }\end{array}$ & .305 & $.740^{\mathrm{ns}}$ \\
\hline Supervisor Evaluation & $\begin{array}{c}5 \text { years } \\
5-9 \text { years } \\
10-14 \text { years } \\
\end{array}$ & $\begin{array}{l}3.73^{\mathrm{a}} \\
3.99^{\mathrm{a}} \\
3.94^{\mathrm{a}} \\
\end{array}$ & $\begin{array}{l}+.59 \\
+.56 \\
\pm .49 \\
\end{array}$ & $\begin{array}{l}\text { Highly Competent } \\
\text { Highly Competent } \\
\text { Highly Competent }\end{array}$ & .624 & $.544^{\mathrm{ns}}$ \\
\hline
\end{tabular}

Means with different letters are significantly different $(\rho=0.05)$. Values are expressed in mean \pm S.D. Symbols and abbreviations: $* *=$ highly significant; * = significant; $\mathrm{ns}=$ not significant

As gleaned from the table above, those teachers with the lowest degree earned also have the lowest evaluation competence. On the other hand, length of service does not appear to have any significant effect on evaluation skills of science teachers. No information or published data has been presented to explain how or why female teachers would have better evaluation skills than male. Several studies have shown that higher educational attainment means better evaluation skills. Kahn and Saeed[32] found out that teachers with higher degree attained have better assessment skills. Goe et al.[33] even highlighted that educational attainment enriches several teacher-evaluation instruments such as (a) classroom observations, (b) instructional artefact, (c) portfolio, (d) teacher-self-report measure, and (e) student survey. With all these literature supports, Jirovec et al.[34] eventually said that teachers' skill in grading was identified as one of the dimensions of teaching effectiveness. 
Table 10. Evaluation skills as affected by trainings and workloads

\begin{tabular}{|c|c|c|c|c|c|c|}
\hline Type of Evaluation & Variable & Mean & S.D. & Descriptive Equivalent & $\mathrm{F}$ & Sig. \\
\hline \multicolumn{7}{|c|}{ Training Points } \\
\hline Self-Evaluation & $\begin{array}{c}<\mathbf{1 0} \text { pts. } \\
11-20 \text { pts. } \\
>20 \text { pts. }\end{array}$ & $\begin{array}{l}\mathbf{2 . 6 9}^{\mathrm{b}} \\
3.60^{\mathrm{a}} \\
3.87^{\mathrm{a}}\end{array}$ & $\begin{array}{l} \pm .55 \\
\pm .51 \\
+.53\end{array}$ & $\begin{array}{c}\text { Competent } \\
\text { Highly Competent } \\
\text { Highly Competent }\end{array}$ & 3.61 & $.019^{*}$ \\
\hline Student Evaluation & $\begin{array}{c}<\mathbf{1 0} \text { pts. } \\
11-20 \text { pts. } \\
>20 \text { pts. }\end{array}$ & $\begin{array}{l}\mathbf{2 . 6 0 ^ { \mathrm { b } }} \\
3.36^{\mathrm{a}} \\
3.62^{\mathrm{a}}\end{array}$ & $\begin{array}{l}+.54 \\
\pm .52 \\
+.50\end{array}$ & $\begin{array}{c}\text { Competent } \\
\text { Highly Competent } \\
\text { Highly Competent }\end{array}$ & 3.24 & $.033^{*}$ \\
\hline Supervisor Evaluation & $\begin{array}{l}<\mathbf{1 0} \text { pts. } \\
11-20 \text { pts. } \\
>20 \text { pts. }\end{array}$ & $\begin{array}{l}3.00^{\mathrm{b}} \\
3.76^{\mathrm{a}} \\
3.89^{\mathrm{a}} \\
\end{array}$ & $\begin{array}{l} \pm .36 \\
\pm .45 \\
\pm .49 \\
\end{array}$ & $\begin{array}{c}\text { Competent } \\
\text { Highly Competent } \\
\text { Highly Competent }\end{array}$ & 3.30 & $.030^{*}$ \\
\hline \multicolumn{7}{|c|}{ Teaching Units } \\
\hline Self-Evaluation & $\begin{array}{l}<20 \text { units } \\
>21 \text { units }\end{array}$ & $\begin{array}{l}3.66^{\mathrm{a}} \\
3.49^{\mathrm{a}} \\
\end{array}$ & $\begin{array}{l} \pm .50 \\
\pm .68 \\
\end{array}$ & $\begin{array}{l}\text { Highly Competent } \\
\text { Highly Competent }\end{array}$ & .543 & $.468^{\mathrm{ns}}$ \\
\hline Student Evaluation & $\begin{array}{l}<20 \text { units } \\
>21 \text { units }\end{array}$ & $\begin{array}{l}3.44^{\mathrm{a}} \\
3.28^{\mathrm{a}} \\
\end{array}$ & $\begin{array}{l} \pm .59 \\
\pm .64 \\
\end{array}$ & $\begin{array}{l}\text { Highly Competent } \\
\text { Highly Competent }\end{array}$ & .501 & $.486^{\mathrm{ns}}$ \\
\hline Supervisor Evaluation & $\begin{array}{l}<20 \text { units } \\
>21 \text { units }\end{array}$ & $\begin{array}{l}3.92^{\mathrm{a}} \\
3.68^{\mathrm{a}} \\
\end{array}$ & $\begin{array}{l} \pm .49 \\
\pm .56 \\
\end{array}$ & $\begin{array}{l}\text { Highly Competent } \\
\text { Highly Competent }\end{array}$ & 1.15 & $.291^{\mathrm{ns}}$ \\
\hline \multicolumn{7}{|c|}{ Number of Preparations } \\
\hline Self-Evaluation & $\begin{array}{c}<2 \text { Prep } \\
2-4 \text { Preps } \\
>5 \text { Preps } \\
\end{array}$ & $\begin{array}{l}3.78^{\mathrm{a}} \\
3.59^{\mathrm{a}} \\
3.47^{\mathrm{a}} \\
\end{array}$ & $\begin{array}{l}+.53 \\
\pm .63 \\
+.51 \\
\end{array}$ & $\begin{array}{l}\text { Highly Competent } \\
\text { Highly Competent } \\
\text { Highly Competent }\end{array}$ & .274 & $.763^{\text {ns }}$ \\
\hline Student Evaluation & $\begin{array}{c}<2 \text { Prep } \\
\text { 2-4 Preps } \\
>5 \text { Preps }\end{array}$ & $\begin{array}{l}3.57^{\mathrm{a}} \\
3.38^{\mathrm{a}} \\
\mathbf{3 . 2 5 ^ { \mathrm { a } }}\end{array}$ & $\begin{array}{l}+.55 \\
\pm .59 \\
+.50\end{array}$ & $\begin{array}{l}\text { Highly Competent } \\
\text { Highly Competent } \\
\text { Competent }\end{array}$ & .322 & $.728^{\text {ns }}$ \\
\hline Supervisor Evaluation & $\begin{array}{c}2 \text { Prep } \\
2-4 \text { Preps } \\
>5 \text { Preps } \\
\end{array}$ & $\begin{array}{l}3.99^{\mathrm{a}} \\
3.81^{\mathrm{a}} \\
3.70^{\mathrm{a}} \\
\end{array}$ & $\begin{array}{l}+.56 \\
\pm .60 \\
+.56 \\
\end{array}$ & $\begin{array}{l}\text { Highly Competent } \\
\text { Highly Competent } \\
\text { Highly Competent }\end{array}$ & .420 & $.662^{\text {ns }}$ \\
\hline \multicolumn{7}{|c|}{ Number of Quasi Assignments } \\
\hline Self-Evaluation & $\begin{array}{l}<2 \text { Quasi } \\
2-4 \text { Quasi } \\
>5 \text { Quasi }\end{array}$ & $\begin{array}{c}3.96^{\mathrm{a}} \\
3.53^{\mathrm{a}} \\
\mathrm{n} / \mathrm{a}\end{array}$ & $\begin{array}{l} \pm .55 \\
\pm .58 \\
\mathrm{n} / \mathrm{a}\end{array}$ & $\begin{array}{l}\text { Highly Competent } \\
\text { Highly Competent } \\
\mathrm{n} / \mathrm{a}\end{array}$ & 1.44 & $.240^{\mathrm{ns}}$ \\
\hline Student Evaluation & $\begin{array}{l}<2 \text { Quasi } \\
2-4 \text { Quasi } \\
>5 \text { Quasi }\end{array}$ & $\begin{array}{c}3.73^{\mathrm{a}} \\
3.33^{\mathrm{a}} \\
\mathrm{n} / \mathrm{a} \\
\end{array}$ & $\begin{array}{c} \pm .53 \\
\pm .57 \\
\mathrm{n} / \mathrm{a} \\
\end{array}$ & $\begin{array}{c}\text { Highly Competent } \\
\text { Highly Competent } \\
\text { n/a }\end{array}$ & 1.41 & $.245^{\mathrm{ns}}$ \\
\hline Supervisor Evaluation & $\begin{array}{l}<2 \text { Quasi } \\
2-4 \text { Quasi } \\
>5 \text { Quasi }\end{array}$ & $\begin{array}{c}3.98^{\mathrm{a}} \\
3.78^{\mathrm{a}} \\
\mathrm{n} / \mathrm{a}\end{array}$ & $\begin{array}{l} \pm .50 \\
\pm .56 \\
\mathrm{n} / \mathrm{a}\end{array}$ & $\begin{array}{l}\text { Highly Competent } \\
\text { Highly Competent } \\
\text { n/a }\end{array}$ & 1.05 & $.313^{\text {ns }}$ \\
\hline
\end{tabular}

Means with different letters are significantly different $(\rho=0.05)$. Values are expressed in mean \pm S.D. Symbols and abbreviations: $* *=$ highly significant; $*$ = significant; $\mathrm{ns}=$ not significant

In Table 10, the impact of trainings and workloads on the evaluation skill competence of science teachers were elucidated. As gleaned from the table, trainings have a significant effect on the evaluation competence of these science teachers. For all the three types of evaluators (self, student and supervisor) it appears that teachers with less than 10 training points are less competent when it comes to their evaluation skills $(2.69 \pm 55,2.60 \pm 42$, and $3.00 \pm 36$, respectively). The number of teaching units and the number of quasi assignments seem not to affect the competence of science teachers. However, it can be observed that the number of preparations of science teachers negatively affect their competence. Based on the student evaluation, those science teachers with more than five preparations are less competent in their evaluation skills $(3.25 \pm 50)$ than those with fewer preparations. It can also be observed that as the number of preparations increases, their competence decreases. Substantial literature indicates the need for training in enhancing evaluation skills of teachers. The study of Rahman et al. [22] showed that training skills of female teachers determined the effectiveness of their use of assessment and evaluation tools. Walls [28] also mentioned that teaching effectiveness is enhanced by having trainings relevant to evaluation and outcomes skills development. Likewise, Okeke [35] said that teacher competence is enhanced through trainings that they have undergone involving evaluation of instructional material programmes. On the other hand, additional workload may negatively affect evaluation skills of teachers as they are obliged to devote less time creating meaningful evaluation and assessment tools compared to those teachers with a smaller number of teaching preparations [36]. Finally, continued personal and institutional development are greatly encouraged to assist teachers improve their competence in the profession and in the society at large [37]. 


\section{Conclusion}

The perceptions on the competence of science teachers along instruction in DMMMSU-NLUC College of Education were evaluated in this study. It was found that trainings have a significant impact on a teacher's personal character, teaching efficiency, general class atmosphere, substantiality of teaching and evaluation skills. Other personal and professional demographic profile were also found to have an effect on the competence of science teachers along instruction. These include the number of children, sex, educational attainment, length of service and workload preparation.

\section{Acknowledgements}

We are grateful to the Don Mariano Marcos Memorial State University for the funding support of the study.

\section{REFERENCES}

[1] Remesh A., "Microteaching, an efficient technique for learning effective teaching," Journal of Research in Medical Sciences: The Official Journal of Isfahan University of Medical Sciences, vol. 18, no. 2, pp. 158-163, 2013. https://www.ncbi.nlm.nih.gov/pmc/articles/PMC3724377/

[2] Goldhaber D. D., Brewer, D. J., "Evaluating the effect of teacher degree level on educational performance," Developments in School Finance, pp. 197-210, 1996. https://eric.ed.gov/?id=ED406400

[3] Bonney E. A., Amoah D. F., Micah S. A., Ahiamenyo C., Lemaire, M. B., "The Relationship between the Quality of Teachers and Pupils Academic Performance in the STMA Junior High Schools of the Western Region of Ghana," Journal of Education and Practice, vol. 6, no. 24, pp.139-150, 2015. https://eric.ed.gov/?id=EJ1078818

[4] Nuangchalerm P, "Inquiry-based learning in China: Lesson learned for school science practices," Asian Social Science, vol. 10, no. 13, pp. 64-71, 2014. https://ssrn.com/abstract $=2459549$

[5] Andersen R., "Reforming Science Teaching: What Research says about Inquiry?," Journal of Science Teacher Education, vol. 13, no. 1, pp. 1-12, 2002. https://doi.org/10.1023/A:1015171124982

[6] Aban J. L., Aban J. L., "Awareness, Perspectives and Practices on the Multifaceted Educational Pedagogies in Don Mariano Marcos Memorial State University, Philippines," International Council of Education for Teaching (ICET), 59th World Assembly - Challenging Disparities in Education. Naruto University of Education, Japan, pp. 447-458, 2015.

[7] Nebeker C., "Basic Research Concepts. San Diego State University Research Foundation."

[8] Babbie E., "The Practice of Social Research," 12th ed Belmont, CA: Wadsworth Cengage, 2010.
[9] Cassel M., Symon S., "Relationships of awareness and practice: Teacher learning in communities," Review of Research in Education, vol. 24, pp. 249-305, 1999.

[10] Cristobal J. M., "Capabilities and needs of the faculty of Lorma Colleges along instruction, research and extension: Basis for a training program," Unpublished Masters Thesis, Saint Louis College, City of San Fernando, La Union, 2004.

[11] Shah S. S. A., "Impact of teacher's behaviour on the academic achievement of university students," Journal of College Teaching and Learning, vol. 6, no. 1, pp. 69-74, 2009. http://prr.hec.gov.pk/jspui/handle/123456789/486

[12] Wenglinsky H., "Teacher classroom practices and student performance: How schools can make a difference," Research Publications Office, Educational Testing Services, Princeton NJ, pp. 1-37, 2001.

[13] Dolev N., Leshem S., "Teachers' emotional intelligence: The impact of training," The International Journal of Emotional Education, vol. 8, no. 1, pp. 75-94, 2016. https://www.um.edu.mt/library/oar/handle/123456789/9996

[14] Jennings P. A., Greenberg M. T., "The pro-social classroom. Teacher social and emotional competence in relation to student and classroom outcomes," Review of Educational Research, vol. 79, no. 1, pp. 491-525, 2009. https://doi.org/10.3102/0034654308325693

[15] Maree J. G., Mokhuane, Q. E., "First steps in developing a community-based teacher training program designed to educate children to be emotionally intelligent," Westport, CT: Praeger, pp. 139-154, 2007

[16] Brackett M. A., Katulak, N. A., "Emotional intelligence in the classroom: A skill-based training for teachers and students," New York: Psychology Press, pp. 1-27, 2006.

[17] Brackett M. A., Alster B., Wolfe C. J., Katulak N. A., Fale, E, "Creating an emotionally intelligent school district: A skill-based approach," Wesport, CT: Praeger, pp. 123-138, 2007.

[18] Brackett M.A., Patti J., Stern R., Rivers S. E., Elbertson N. A., Chrisholm C. and Salovey P., "A sustainable, skill-based approach to building emotionally literate schools," San Francisco, CA: Pfeiffer, pp. 329-358, 2009.

[19] Wilson S. M., Floden R. E., Ferrini-Mundy J., "Teacher preparation research: An insider's view from the outside." Journal of Teacher Education, vol. 53, no. 2, pp. 147-158, 2002. https://doi.org/10.1177/0022487102053003002

[20] Tyagi S., "A study of teaching effectiveness of secondary school teachers in relation to their demographic characteristics," International Journal of Engineering and Innovative Technology, vol. 3, no. 1, pp. 288-295, 2013.

[21] Centra J. A., "Reflective faculty evaluation: Enhancing teaching and determining faculty effectiveness," San Francisco: Jossey-Bass, pp. 44-98, 2003.

[22] Rahman F., Jumani N. B., Akhter Y., "Relationship between training of teachers and effectiveness teaching," International Journal of Business and Social Science, vol. 2, no. 4, pp. 23-38, 2011.

[23] Wenglinsky H., "The link between teacher classroom practices and student academic performance," Education 
Policy Analysis Archives, vol. 10 no. 12, pp. 1-22, 2002 https://epaa.asu.edu/ojs/article/view/291

[24] Wayne A. J., Young, P., "Teacher characteristics and student achievement gains: A review," Review of Educational Research, vol. 73, no. 1, pp. 89-122, 2003. https://doi.org/10.3102/00346543073001089

[25] Bill G. M. "Student perceptions and the MET project measures of effective teaching project," Review of Educational Research, vol. 22, pp. 169-174, 2010.

[26] Delaney J. G., "How high school students perceive effective teachers," Faculty of Education, Memorial University of Newfoundland, pp 3-7, 2010.

[27] Stronge J. H., "Qualities of effective teachers," Alexandria, VA: Association for Supervision and Curriculum Division, pp. 189-232, 2002.

[28] Walls R. T., "Psychological foundations of learning," Morgantown, WV: WVU International Centre for Disability Information, pp. 12-37, 1999.

[29] Thoreson C., "Teachers competence and evaluation of instructional materials programmes in Austere time," Ibadan, Y-books makers Nig. Ltd, pp. 231-239, 2007.

[30] Colfalter C., Ladd H., Vigdor J., "How and why do teachers' credentials matter for students' achievement?," Memeo Duke University, pp. 15-37, 2006.

[31] Ukewe I., "The impact of teachers' qualification and school facilities on the academic performance of students in physical education in two states," Unpublished. M. Ed. Thesis, University of Calabar, pp. 1-210, 1999.

[32] Khan S. H., Saeed, M., "Effectiveness of pre-service teacher education programme in Pakistan," Journal of Pakistani Education, vol. 31, no. 1, pp. 83-98, 2009.

[33] Goe L., Bell C., Little O., "Approaches to evaluating teacher effectiveness, a research synthesis," Washington D.C. National Comprehensive Centre for Teacher Quality, pp. 6-13, 2008.

[34] Jirovec R. L., Ramanthan C. S., Alvarez A. R. "Course evaluations: What are social work students telling about teaching effectiveness?," Journal of Social Work Education, vol. 34, pp. 229-236, 1998. https://doi.org/10.1080/1043779 7.1998.10778919

[35] Okeke T. O., "Trends and research in educational technology," Ibadan, Y-books makers Nig. Ltd, pp. 78-139, 2003.

[36] Ukwayi G. U., "Motivation, job satisfaction and attitude to work among secondary school teachers in two states," Unpublished M. Ed. Thesis, University of Calabar, pp. 1-323, 2001.

[37] Gepila E. Jr., "Assessing teachers using Philippine Standards for Teachers," Universal Journal of Educational Research, vol. 8, no. 3, pp. 739 - 746, 2020. DOI: 10.13189/ujer.2020. 080302 . 